\title{
Mithramycin encapsulated in polymeric micelles by microfluidic technology as novel therapeutic protocol for beta-thalassemia
}

This article was published in the following Dove Press journal:

International Journal of Nanomedicine

17 January 2012

Number of times this article has been viewed

\author{
Lorenzo Capretto' \\ Stefania Mazzitelli² \\ Eleonora Brognara ${ }^{2}$ \\ Ilaria Lampronti² \\ Dario Carugo' \\ Martyn Hill' \\ Xunli Zhang' \\ Roberto Gambari² \\ Claudio Nastruzzi ${ }^{3}$ \\ 'Engineering Sciences, University \\ of Southampton, Southampton, UK; \\ ${ }^{2}$ Department of Biochemistry and \\ Molecular Biology, ${ }^{3}$ Department of \\ Pharmaceutical Sciences, University of \\ Ferrara, Ferrara, Italy
}

Correspondence: Claudio Nastruzzi Department of Pharmaceutical Sciences, University of Ferrara, Via Fossato di Mortara 17/I9, Ferrara, 44I2I, Italy Tel +390532455255

Fax +390532455953

Email nas@unife.it

\begin{abstract}
This report shows that the DNA-binding drug, mithramycin, can be efficiently encapsulated in polymeric micelles (PM-MTH), based on Pluronic ${ }^{\circledR}$ block copolymers, by a new microfluidic approach. The effect of different production parameters has been investigated for their effect on PM-MTH characteristics. The compared analysis of PM-MTH produced by microfluidic and conventional bulk mixing procedures revealed that microfluidics provides a useful platform for the production of PM-MTH with improved controllability, reproducibility, smaller size, and polydispersity. Finally, an investigation of the effects of PM-MTH, produced by microfluidic and conventional bulk mixing procedures, on the erythroid differentiation of both human erythroleukemia and human erythroid precursor cells is reported. It is demonstrated that PM-MTH exhibited a slightly lower toxicity and more pronounced differentiative activity when compared to the free drug. In addition, PM-MTH were able to upregulate preferentially $\gamma$-globin messenger ribonucleic acid production and to increase fetal hemoglobin ( $\mathrm{HbF}$ ) accumulation, the percentage of $\mathrm{HbF}$-containing cells, and their $\mathrm{HbF}$ content without stimulating $\alpha$-globin gene expression, which is responsible for the clinical symptoms of $\beta$-thalassemia. These results represent an important first step toward a potential clinical application, since an increase in $\mathrm{HbF}$ could alleviate the symptoms underlying $\beta$-thalassemia and sickle cell anemia. In conclusion, this report suggests that PM-MTH produced by microfluidic approach warrants further evaluation as a potential therapeutic protocol for $\beta$-thalassemia.
\end{abstract}

Keywords: microfluidics, lab-on-a-chip, design of experiments, erythroid differentiation, human erythroid precursor cells

\section{Introduction}

Mithramycin A (MTH), also called plicamycin, is an aureolic acid-type polyketide, which is normally isolated from various strains of the bacterium Streptomyces. ${ }^{1}$ MTH has been used clinically for many years to treat testicular carcinoma and several types of cancer including leukemia, as well as hypercalcemia in patients with metastatic bone lesions and Paget's disease. ${ }^{2}$ The ability of MTH to bind the DNA minor groove is of great interest, since pharmacologically-mediated modulation of DNA/nuclear protein complex formation represents a promising approach to control gene expression. For instance, MTH was found to inhibit the binding of transcription factor Sp1 to its promoter, ${ }^{3}$ leading to gene transcription modulation of different genes, including $V E G F^{4} c-M Y C$, and $h a-R A S$, as well as antiapoptotic genes. ${ }^{5}$ It was also shown that MTH inhibited p53-mediated transcriptional responses, DNA methyltransferase, and the multidrug resistant gene $M D R 1,{ }^{6}$ therefore putatively sensitizing tumor cells to chemotherapeutic agents. 
Furthermore, because of its ability to bind DNA, MTH has been proposed as a potential therapeutic agent for the treatment of hematological diseases such as $\beta$-thalassemia and sickle cell anemia. In these pathological cases, the rationale for using MTH relies on the pharmacologicallymediated upregulation of the expression of human $\gamma$-globin genes. ${ }^{7,8}$ For instance, it has been demonstrated that MTH can induce erythroid differentiation of the human leukemic K562 cell line through the induction of $\gamma$-globin messenger ribonucleic acid (mRNA) accumulation. This data is particularly important for clinic applications since even a moderate increase in the production of fetal hemoglobin $(\mathrm{HbF})$ could be associated with a significant improvement in the clinical status of the patients. ${ }^{9}$ Moreover, it should be noted that, at least in vitro, the differentiative activity is achieved at lower concentrations with respect to those required for antiproliferative action. ${ }^{8}$

In spite of the above mentioned therapeutic potentials, the clinical use of MTH, especially as anticancer drug that requires higher doses, is still limited, mainly due to its severe side effects including gastrointestinal, hepatic, kidney, and bone marrow toxicity. ${ }^{10}$ One of the possible strategies to enhance its safety and efficacy is to develop different formulations of delivery systems such as nanoparticles or microparticles, ${ }^{11}$ liposomes, ${ }^{12}$ and polymeric micelles (PMs). ${ }^{13}$ PMs are a class of polymeric nanoparticles with a core-shell structure that form spontaneously by self-assembly of amphiphilic block copolymer unimers in water. Unimer self-assembly occurs as a result of hydrophobic or electrostatic interactions between polymer segments. ${ }^{14}$ The development of a delivery system for MTH based on PMs could result in a series of advantages including controlled delivery, alteration of drug circulation time, changes in cellular distribution, and increase in amount of drug delivered to target cells. Taken together, the use of PMs has been demonstrated to result in higher drug efficacy and lower side effects. ${ }^{15}$

In particular, the drug circulation time is increased by the presence of the polyethylene glycol shell. Polyethylene glycol chains create a highly water-bound barrier on the particle surface which blocks the adhesion of opsonins and the subsequent recognition and phagocytosis by the reticuloendothelial system. ${ }^{16}$ Circulation time is additionally prolonged by reduced renal excretion of PMs compared to free drug. ${ }^{17}$ A prolonged circulation time is particularly beneficial when the target of drug action is represented by circulating and bone marrow cells, as in the case of erythroid differentiating drugs. ${ }^{18}$ Another advantage of PMs that could be important for differentiating drugs is related to the enhanced permeation retention effect. ${ }^{19}$ This effect favors the accumulation of nanoparticles in tissues characterized by increased vascular permeability and impaired lymphatic drainage. ${ }^{20}$ The long circulating properties of PMs have been investigated by many authors that have demonstrated the potential of PMs as delivery system for different anticancer drugs including taxanes and doxorubicin. ${ }^{21-23}$ Several PM formulations have entered clinical trials for the treatment of tumors refractory to conventional treatments ${ }^{24}$ and colon and gastric cancers. ${ }^{25}$

The innovation in PM technology resides in a number of features that make them one of the most promising drug delivery systems. On the one hand, when compared to microparticle-based formulations, the nanoscale dimensions of PMs permit their intravenous administration and allow them to freely circulate in the blood stream. ${ }^{20}$ On the other hand, when compared to liposomes, which represent an example of nanoscale formulations (in this case constituted of low molecular weight amphiphils), PMs exhibit greater resistance to dissociation upon dilution into the blood stream. This advantage is particularly beneficial in reducing drug leakage from the delivery system. ${ }^{21}$

PMs represent an appealing formulation for MTH since the drug molecule presents specific attributes fitting the PMs' nanoenvironment, which is characterized by a hydrophobic core and an outer hydrophilic shell. MTH has lipophilic moieties in its molecular structure; in this respect, MTH is relatively soluble in water, acetone, ethyl acetate, and methyl isobutyl ketone and soluble in lower alcohols and dimethyl sulfoxide (DMSO); it is slightly soluble in diethyl ether and benzene and insoluble in petroleum ether, carbon tetrachloride, and cyclohexane. MTH shows a $\log P$ of $1.290 \pm 1.454,{ }^{26}$ suggesting a slight lipophilic nature and the potential to be associated in PMs. It has been demonstrated that the equilibrium partition coefficients of a drug between micelles and water strongly correlate with the drug's octanol/ water partition coefficient. ${ }^{27}$

With respect to $\mathrm{PM}$ preparation, currently two main approaches are usually followed depending on the physicochemical properties of block copolymer(s) and drug. ${ }^{28}$ One method is based on the direct dissolving of the polymer together with the drug in an aqueous environment, usually involving moderately hydrophilic copolymers (eg, poloxamers) that are readily soluble in water. By contrast, the second approach is relevant for copolymers that are sparingly soluble in water, thus a water miscible organic solvent is needed to dissolve both copolymer and drug. The commonly used solvents include DMSO, N,N-dimethylformamide, 
acetonitrile, tetrahydrofuran, acetone, and dimethylacetamide. Subsequently, the solvents are generally removed by "bulk" procedures such as dialysis against water. The solvent removal by dialysis is usually widespread even if it may not be readily transposable to the industrial scale. ${ }^{29}$ In this method, solvent removal is particularly crucial since it triggers the nanoprecipitation process and therefore the final PM dimensional and entrapment characteristics.

Recently, an innovative and highly controlled procedure for PM preparation based on a microfluidic approach was described. ${ }^{30}$ This protocol allows the removal of organic solvent in a controlled fashion, through a fast and adjustable mixing process made possible by the peculiar microfluidic environment characteristics. By this method, the production of PMs is robust, reproducible, and permits precise control of PM physicochemical characteristics, essential for their biopharmaceutical applications. With respect to conventional bulk methods, PMs produced by microfluidic reactors show a number of advantages, including: smaller mean size, high homogeneity, and increased drug loading efficiency. ${ }^{30}$ Regarding PM size and size distribution, previous studies demonstrated that small PMs are more effective at evading scavenging from macrophages, exhibiting prolonged circulation in the blood stream. ${ }^{31}$ In addition, microfluidics does not require postprocessing steps, which are normally needed for "bulk" procedure to remove large particles or aggregates, before their in vitro or in vivo testing. ${ }^{32}$

The current study presents the development of an advanced formulation for mithramycin encapsulated in polymeric micelles (PM-MTH) produced by microfluidic technology and in vitro analysis of the formulation as a new therapeutic protocol for $\beta$-thalassemia. For comparison, PMs produced with conventional bulk methods were also considered. The production parameters and their effects on PM characteristics were analyzed, and the effects of MTH association in PMs on the erythroid differentiation of both human erythroleukemia (K562) and human erythroid precursor cells (ErPC) were investigated. For the production of PM-MTH, Pluronic ${ }^{\circledR}$ F127 (BASF Chem Trade GmbH, Burgbernheim, Germany) was employed as a model block copolymer, due to its well-studied characteristics and the impressive safety profile that makes it Food and Drug Administration approved for pharmaceutical and medical applications, including parenteral administration. ${ }^{33}$

Taking into account the combination of advantages and possible complications and side effects of PMs, poly(ethylene oxide)-poly(propylene oxide) block copolymer micelles represent an appealing alternative to other advanced drug delivery formulations, especially for drugs exhibiting a limited solubility in water. Future perspectives for their approval as drug formulations for human use will still need to overcome a number of drawbacks, the most significant of which appears to be the low stability of the self-assembled nanostructures upon dilution in the bloodstream.

\section{Materials and methods Materials}

The polymer Pluronic ${ }^{\circledR}$ F127 (Lutrol $^{\circledR}$ F127) was provided, as a kind gift, by BASF Chem Trade GmbH. DMSO and MTH were obtained from Sigma-Aldrich (Dorset, United Kingdom).

\section{Fabrication of microfluidic reactors}

The glass-made microfluidic reactors employed for the preparation of PMs were fabricated by a photolithography/wet etching procedure, as previously reported. ${ }^{34}$ Firstly, the channel pattern was designed by AutoCAD ${ }^{\circledR}$ drawing software (Autodesk, Inc, San Rafael, CA), thereafter, a negative film representing the optical mask was obtained by a commercial photomask producer (JD Photo-Tools, Oldham, Lancashire, United Kingdom). Crown white glass (B-270) plates (thickness of $1.5 \mathrm{mM}$ ) coated with a thin layer of chromium metal mask plus an upper layer of positive photoresist (AZ 1500; Telic Company, Valencia, CA) were used for channel network fabrication. The channel pattern was transferred by ultraviolet exposure from the negative film to the photoresist layer on the glass sheet. After the pattern transfer, the glass plate was baked in an oven at $80^{\circ} \mathrm{C}$ overnight ( $\sim 17$ hours) to dry and harden the mask on glass. The channels were then etched using $1 \%$ hydrofluoric acid (Sigma-Aldrich) buffered with 5\% ammonium fluoride (Sigma-Aldrich) solution at $65^{\circ} \mathrm{C}$ under ultrasonic agitation (Ultrasonic Bath; VWR International, Lutterworth, Leicestershire, United Kingdom). Finally, the etched glass was thermally bonded $\left(595^{\circ} \mathrm{C}\right.$ for 3 hours) to a top glass plate into which outlet and inlet holes had been previously drilled to link the channels. Teflon tubes (Upchurch Scientific, Oak Harbor, WA) with an inner diameter of $500 \mu \mathrm{m}$ were used to connect the reactor to gastight glass syringes (Hamilton Company, Reno, NV) controlled by a KDS syringe pump (KD100; KD Scientific Inc, Holliston, MA) for reagent delivery.

\section{Preparation of PMs in microfluidic reactor and by bulk mixing}

PMs were prepared by the microfluidic approach using a microfluidic reactor consisting of three inlets, one main 
channel, and a single outlet (Figure 1). The channel architecture allowed the creation of a hydrodynamic flow focusing configuration. The focusing was generated where the central stream of polymer/drug/DMSO solution met the two lateral sheath streams of water side-by-side. A photomicrograph of the typical arrow shape liquid focusing is shown in the upper inset of Figure 1. The volumetric ratio of the organic solution over water $(R)$ was varied by changing the relative flow rates. The dimensions (in $\mathrm{mM}$ ) of the microfluidic reactor are reported in Figure 1. All channels had a depth of $30 \mu \mathrm{m}$. The channels had a semicircular cross-sectional shape so that channel width was strictly connected with channel depth, due to the isotropicity of the fabrication procedure. ${ }^{34,35}$ The flow within

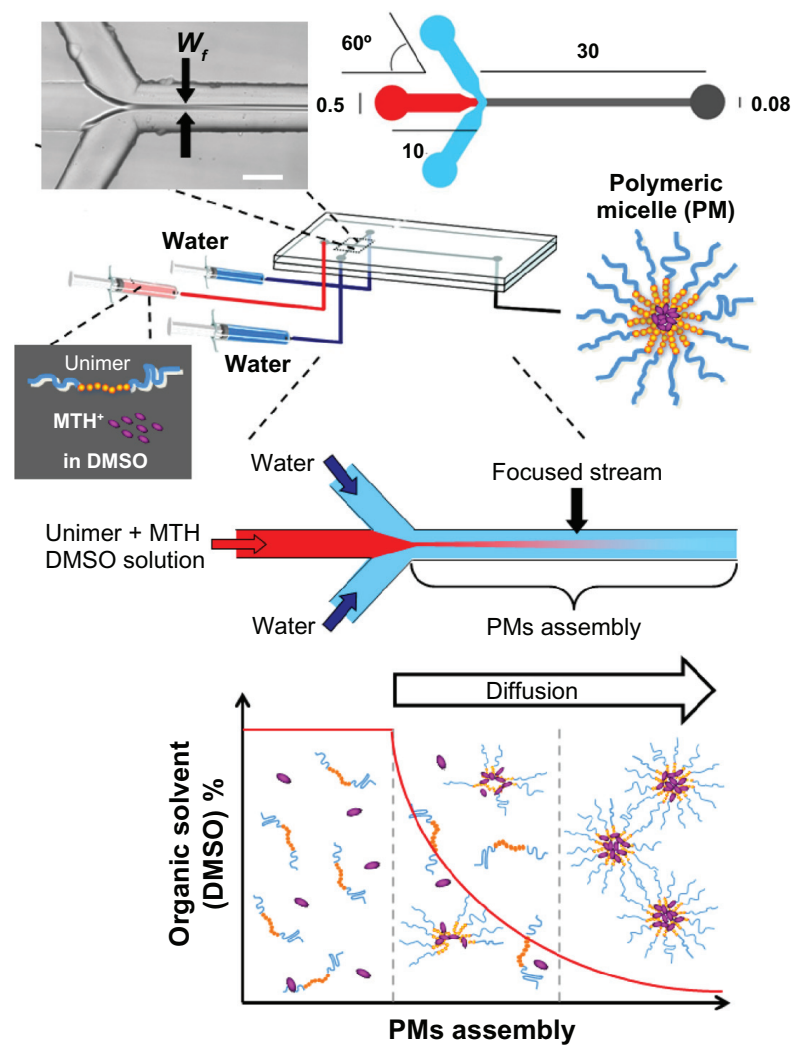

Figure I Schematic representation of the preparation procedure for polymeric micelles by microfluidics. $W_{f}$ represents the width of the focused stream generated when the central stream of Pluronic ${ }^{\circledR}$ FI 27 (BASF Chem Trade GmbH, Burgbernheim, Germany) (unimers) and mithramycin dissolved in dimethyl sulfoxide and the two lateral sheath streams of water met at the channel junction. The right upper drawing reports the dimensional characteristics of the glass microfluidic device. The lower graph depicts the formation mechanism of mithramycin encapsulated in polymeric micelles in microfluidic environment, showing how the progressive mutual diffusion of dimethyl sulfoxide and water molecules causes the unimers to orientate themselves so that the hydrophobic blocks are removed from the aqueous environment forming the primary polymeric micelle's nuclei. As the dimethyl sulfoxide/water diffusion proceeds, polymeric micelles grow to their final structure and size (see detailed explanation in results and discussion section).

Abbreviations: DMSO, dimethyl sulfoxide; MTH, mithramycin; PM, polymeric micelle. the microchannels was monitored by an inverted microscope (IX51; Olympus Corporation, Tokyo, Japan). PMs were also prepared by bulk mixing. A polymeric solution in DMSO, in the presence or absence of MTH, was added to $2-10 \mathrm{~mL}$ of deionized water in a glass round-bottom flask and thoroughly mixed by vortexing for 5 minutes. Different volumetric ratios $(\mathrm{R})$ of organic solution to water were selected varying between 0.03 and 0.13 . For both microfluidic and bulk mixing procedures, the preparation of PMs was performed at environmental temperature, typically ranging between $21^{\circ} \mathrm{C}-23^{\circ} \mathrm{C}$.

\section{Characterization of PMs}

Before characterization, the amount of water was adjusted in order to have the same DMSO/water ratio in all samples (ie, 3.2\% DMSO volume/volume).

The size and size distribution of PMs were determined by dynamic light scattering (Zetasizer Nano ZS; Malvern Instruments Ltd, Malvern, Worcestershire, United Kingdom) and/or by nanoparticle tracking analysis (LM10; NanoSight Ltd, Amesbury, Wiltshire, United Kingdom). For each measurement, $300 \mu \mathrm{L}$ of PM suspension was loaded in the detection cell and five measurements were performed to give an average value using Zetasizer Software 6.12 (Malvern Instruments) and nanoparticle tracking analysis (NTA) system NanoSight LM10 (NanoSight Ltd, Amesbury, UK). PM sizing was performed at environmental temperature, typically ranging between $21^{\circ} \mathrm{C}-23^{\circ} \mathrm{C}$.

To estimate the amount of drug associated in small PMs (ie, mean diameter $\leq 200 \mathrm{~nm} ; \mathrm{PMs}_{200}$ ) and large PMs (ie, mean diameter $\geq 200 \mathrm{~nm}$ ), PM suspension, after preparation, was passed through a $0.2 \mu \mathrm{m}$ pore size filter, as previously reported. ${ }^{36}$ MTH concentration in filtrates was estimated by measuring its fluorescence at $\lambda_{\mathrm{ex}}=440 \mathrm{~nm}$ and $\lambda_{\mathrm{em}}=523 \mathrm{~nm}$ using a Cary Eclipse Fluorescence Spectrophotometer (Agilent Technologies, Stockport, United Kingdom). The percentage of drug associated in $\mathrm{PMs}_{200}\left(\operatorname{drug}_{200}\right)$ was calculated using the following equation:

$$
\operatorname{Drug}_{200}=\mathrm{a} / \mathrm{b} \times 100
$$

where $\mathrm{a}$ is the weight amount of drug in $\mathrm{PMs}_{200}$ and $\mathrm{b}$ is the total amount of drug used for PM preparation.

To estimate the drug association, defined as the percentage of PM-MTH, samples were ultrafiltrated through a semipermeable membrane with 3000 Da nominal molecular weight limit (Amicon ${ }^{\circledR}$ Ultra-4 Centrifugal Filter Unit; Millipore Ltd, Watford, United Kingdom) for 15 minutes at $1.25 \mathrm{~g}$, to separate water phase from 
micelles. The amount of free MTH in the ultrafiltrate was determined by spectrofluorimetric analysis at $\lambda_{\mathrm{ex}}=440 \mathrm{~nm}$ and $\lambda_{\mathrm{em}}=523 \mathrm{~nm}$.

\section{Experimental design and statistics}

The influence of experimental parameters on the PM size and drug $_{200}$ was studied by design of experiments (DoE) approach consisting of eleven runs. The experimental design and evaluation of the experiments were performed by the software MODDE 8.0 (Umetrics AB, Umea, Sweden), followed by multiple linear regression algorithms, as previously described. ${ }^{37}$

\section{K562 cells}

K562 cells were cultured in humidified atmosphere of $5 \%$ carbon dioxide in Roswell Park Memorial Institute-1640 medium (Sigma-Aldrich) supplemented with 10\% fetal bovine serum (Celbio SpA, Milan, Italy), $50 \mathrm{U} / \mathrm{mL}$ penicillin, and $50 \mu \mathrm{g} /$ $\mathrm{mL}$ streptomycin. Treatment of K562 cells with free MTH (dissolved in DMSO at the appropriate concentrations) or PMMTH was carried out by adding the appropriate concentration of drug at the beginning of the cultures (cells were seeded at $30,000 / \mathrm{mL}$ ). The medium was not changed during the entire induction study. The proportion of benzidine-positive cells (which contain hemoglobin, an index of erythroid differentiation) was determined after 7 days in culture using a solution containing $0.2 \%$ benzidine in $5 \mathrm{M}$ glacial acetic acid $(10 \%$ hydrogen peroxide), as previously described. ${ }^{8}$

\section{ErPC cultures}

Patients were from Thalassemic Day Hospital, S Anna Hospital, University of Ferrara, Ferrara, Italy; written informed consent was obtained from each patient and the samples of peripheral blood were collected just before the transfusion treatment. Mononuclear cells were isolated by Ficoll-Hypaque density gradient centrifugation and seeded in $\alpha$-minimal essential medium supplemented with $10 \%$ fetal bovine serum (Celbio), $1 \mu \mathrm{g} / \mathrm{mL}$ cyclosporine A (Sandoz, Basel, Switzerland) and 10\% conditioned medium from the 5637 bladder carcinoma cell line. The cultures were incubated at $37^{\circ} \mathrm{C}$, under an atmosphere of $5 \%$ carbon dioxide in air with extra humidity. After 7 days incubation in this phase I culture, the nonadherent cells were harvested, washed, and then cultured in fresh medium composed of $\alpha$-medium, $30 \%$ fetal bovine serum, $1 \%$ deionized bovine serum albumin, $10^{-5} \mathrm{M} \beta$-mercaptoethanol, $1.5 \mathrm{mM} \mathrm{L}$-glutamine, $10^{-6} \mathrm{M}$ dexamethasone, $1 \mathrm{U} / \mathrm{mL}$ human recombinant erythropoietin (Tebu-bio, Milan, Italy), and stem cell factor (Inalco, Milan,
Italy). This part of the culture is referred to as phase II. After phase II, the induction period with erythroid inducer MTH was usually 4 days.

\section{RNA extraction and real-time quantitative reverse transcription- polymerase chain reaction (RT-PCR)}

Cells were isolated by centrifugation at $1500 \mathrm{rpm}$ for 10 minutes at $4^{\circ} \mathrm{C}$, washed in phosphate buffered saline, and lysed in TRI Reagent ${ }^{\circledR}$ TM (Sigma-Aldrich), according to the manufacturer's instructions. The isolated RNA was washed once with cold $75 \%$ ethanol, dried, and dissolved in diethylpyrocarbonate-treated water before use. RT-PCR was performed as follows. After production of complementary DNA, using $1 \mu \mathrm{g}$ total RNA, a control PCR for $\gamma$-globin gene expression was performed using the $\gamma$-globin mRNAspecific primers 5'-ACTCGCTTCTGGAACGTCTGA-3' and 5'-AGTGCCCTGTCCTCCAGATAC-3'. Quantitative realtime PCR assay of $\gamma$-globin mRNA, $\beta$-globin, and $\alpha$-globin transcripts were carried out using gene-specific double fluorescent-labeled probes in a 7700 Sequence Detection System version 1.6.3 (Applied Biosystems, Warrington, Cheshire, United Kingdom). The following primer and probe sequences were used for real-time PCR: $\gamma$-globin forward primer, 5'-TGGCAAGAAGGTGCTGACTTC-3'; $\gamma$-globin reverse primer, 5'-TCACTCAGCTGGGCAAAGG-3'; $\gamma$-globin probe, 5'-FAM-TGGGAGATGCCATAAAGCACCTGG TAMRA-3'; $\beta$-globin forward primer, 5'-CAAGAAAGT GCTCGGTGCCT-3'; $\beta$-globin reverse primer, 5'-GCAAAGGTGCCCTTGAGGT-3'; $\beta$-globin probe, 5'-FAM-TAGTGATGGCCTGGCTCACCTGGAC TAMRA-3'; $\alpha$-globin forward primer, 5'-TCCCCACCAC CAAGACCTAC-3'; $\alpha$-globin reverse primer, 5'-CCTTAACCTGGGCAGAGCC-3'; $\alpha$-globin probe, 5'-FAM-TCCCGCACTTCGACCTGAGCCA TAMRA- $3^{\prime}$. The fluorescent reporter and the quencher were 6-carboxyfluorescein (FAM) and 6-carboxy- $\mathrm{N}, \mathrm{N}, \mathrm{N}^{\prime}, \mathrm{N}^{\prime}$ tetramethylrhodamine (TAMRA), respectively. For real-time PCR of the reference genes, the endogenous control human glyceraldehyde-3-phosphate dehydrogenase was used, and $\beta$-actin kits and the probes were fluorescently labeled with Taqman ${ }^{\circledR}$ VIC dye (Applied Biosystems).

\section{High performance liquid chromatography (HPLC) analysis of hemoglobin}

ErPC were harvested, washed once with phosphate buffered saline, and the pellets were lysed in lysis buffer (sodium 
dodecyl sulfate $0.01 \%$ ). After incubation on ice for 15 minutes, and spinning for 5 minutes at 14,000 rpm in a microcentrifuge, the supernatant was collected and injected. Hemoglobin present in the lysate was separated by cation-exchange HPLC, ${ }^{38}$ using a System Gold 126 Solvent Module-166 Detector (Beckman Coulter Ltd, High Wycombe, United Kingdom). Hemoglobin was separated using a Syncropak CCM 103/25 (250 mM × $4.6 \mathrm{mM})$ column (Rotork, Bath, United Kingdom), samples were eluted in a solvent gradient using aqueous sodium acetate-Bis Tris-potassium cyanide buffers, and detection was performed at $415 \mathrm{~nm}$. The standard controls were purified adult hemoglobin (Sigma-Aldrich) and $\mathrm{HbF}$ (Alfa Wassermann, Milan, Italy).

\section{Statistical data analysis}

All the data were normally distributed and presented as mean \pm standard deviation. Statistical differences between groups were compared using one-way analysis of variance between groups. Statistical significance was assumed at $P$ values less than 0.05 or 0.001 .

\section{Results and discussion Production of PMs by microfluidics: general considerations and mechanism of PM formation}

Nanotechnology has considerable potential for the treatment of many diseases, due to the opportunity of nanocarriers (ie, PMs) to control the temporal and spatial distribution of drugs within different organs or tissues. However, nanocarriers for drug delivery require a number of specific requisites, such as defined size and size distribution, depending on their administration route and therapeutic application. ${ }^{39}$

In this respect, a series of experiments was started, aimed at the production of precisely size controlled PMs by microfluidic approach. The procedure, developed in the authors' laboratory, is reported in Figure 1, together with a scheme of the employed microfluidic reactor, the experimental setup, and a drawing illustrating the mechanism of PM formation.

In the preliminary step of the procedure, polymer and drug are dissolved in DMSO, in which both species are molecularly dispersed as either single polymer unimers or drug molecules, respectively. The DMSO solution is then injected into the microfluidic device where the entire formation process (unimer self-assembly in PMs) occurs. The employed microfluidic device is characterized by a hydrodynamic flow focusing configuration (Figure 1) in which the central stream of polymer and drug in DMSO solution flowing along the main channel is hydrodynamically focused when it meets two lateral sheath streams of water side-by-side.

As DMSO comes in contact with water, the mutual diffusion of DMSO and water molecules causes a progressive increase of the microenvironment hydrophilicity in which the unimers and drug reside (ie, due to water content rise). As a consequence of the microenvironment change, the unimers orientate themselves so that the hydrophobic blocks are removed from the aqueous environment in order to achieve a state of minimum free energy. ${ }^{17,39}$ This process causes the nucleation of unimers forming the primary PM's nuclei. ${ }^{40} \mathrm{It}$ is likely that at this stage MTH molecules move towards the nascent PMs, which represent an environment suitable for MTH solubilization, as proved by the MTH partition water/ octanol partition coefficient $(\log P=1.290 \pm 1.454) .{ }^{26}$ As the DMSO/water diffusion proceeds, in parallel, PMs grow to their final structure and size. ${ }^{17}$

The reciprocal diffusion of solvent molecules represents the crucial step of micelle formation, thereby controlling the self-assembly process and size characteristics of the produced PMs. For instance, it has been demonstrated that slowing the rate of organic solvent removal led to larger and polydispersed PMs. ${ }^{40}$ On the other end, a fast and uniform solvent displacement, achievable in microfluidic device, is beneficial in controlling the self-assembly process, leading to small PMs with a narrow size distribution. ${ }^{30}$ It is noteworthy that, within the microfluidic device, the organic solvent (ie, DMSO) removal process is mainly controlled by the width of the focused stream $\left(W_{f}\right)$, which is in turn controlled by the volumetric flow ratio between central (ie, DMSO solution) and sheath (ie, water) streams.

With respect to the choice of amphiphilic polymer for the production of PMs, the well-known Pluronic F127 polymer was chosen on the basis of the following consideration. Pluronic block copolymers can be suitably employed for the production of the drug delivery system with multiple effects. ${ }^{41}$ The incorporation of drugs into PMs formed by Pluronic results in enhanced metabolic stability and circulation time, with beneficial effects in increasing drug efficacy and lowering side effects. In addition, Pluronic F127, thanks to its well-studied characteristics and impressive safety profile, was Food and Drug Administration approved for pharmaceutical and medical applications, including parenteral administration. ${ }^{33}$

Keeping in mind all these considerations, the initial part of the current study was devoted to the investigation of the experimental conditions influencing the size 
and size distribution of the produced PMs. The analysis was performed by a changing-one-separate-factor-a-time (COST) approach, which represents an intuitive method of experimental design involving the testing of factors, or causes, one at a time instead of all simultaneously. Prominent text books and academic papers currently favor factorial experimental designs, where multiple factors are changed at once. ${ }^{42,43}$ Despite this criticism, some researchers have demonstrated that the practical information you can obtain by COST can be later employed for a successive DoE. In fact, informative DoE results are generally obtained when there is good knowledge of the investigated system. COST approach permits the selection of the factors that affect the process and the reduction of process variability. ${ }^{43}$

It should be also noted that, from a practical point of view, the experimental approach based on microfluidics is intrinsically simple and mainly governed by few experimental parameters (factors). The factors involved in the production of PMs by microfluidics are generally limited to: (a) size of the channel of the microfluidic device, (b) total flow rate, (c) volumetric ratio $(\mathrm{R})$ between polymeric solution and water, (d) concentration of polymer, and (e) concentration of drug. ${ }^{30,32}$ With respect to channel size and total flow rate, previous investigations have demonstrated that it is possible to produce PMs in a robust and reproducible fashion when using a microfluidic device similar in size (channels) to that employed for the present investigation and a total flow rate of $0.65 \mathrm{~mL} / \mathrm{minute}^{44}$

For these reasons, attention was focused on a reduced number of experimental parameters that are most likely to influence PM-MTH self-assembly, including volumetric ratio $\mathrm{R}$ and polymer and MTH concentration.

\section{Effects of microfluidic parameters on PM characteristics}

As a first parameter, the volumetric flow ratio $\mathrm{R}$ was considered. It represents the volumetric ratio between the liquid flowing in the central channel (polymer/drug/DMSO) and that flowing in the two lateral channels (water). $\mathrm{R}$ is strictly related to the mixing time between solvent/nonsolvent (DMSO/water) which represents the key step in the production of PMs by microfluidics. ${ }^{30}$ The effect of $\mathrm{R}$ was analyzed by varying its values in the range between 0.03 and 0.13. Pluronic F127 concentration within the DMSO inlet stream was set at $7.5 \mathrm{mM}$. The results reported in Figure 2 shows that PMs produced by microfluidic reactor have a mean radius $52-61 \mathrm{~nm}$, and the $\mathrm{R}$ value positively correlates with PM size. For comparison, the dimensional
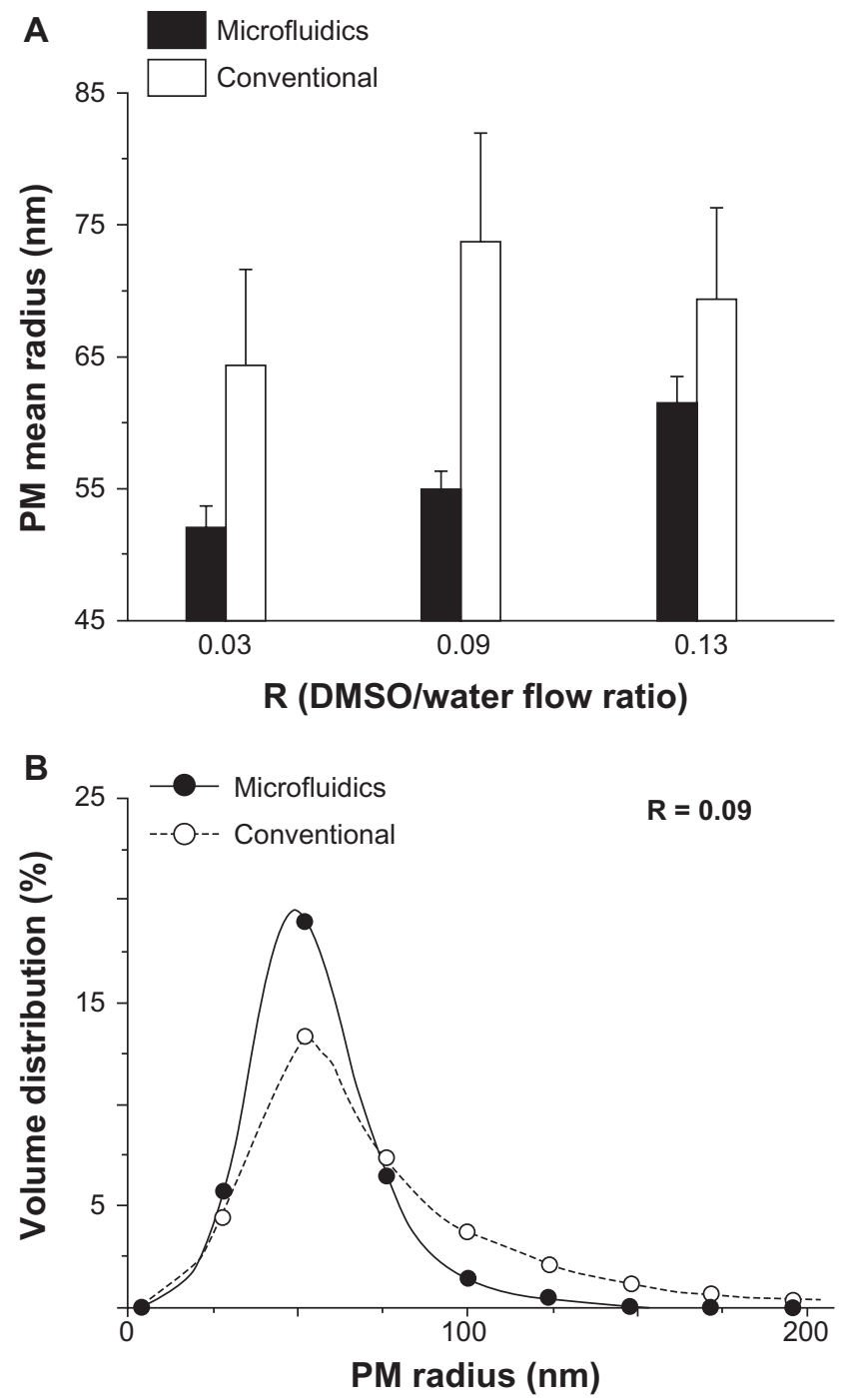

Figure 2 Effect of the volumetric ratio between dimethyl sulfoxide (solubilizing Pluronic $^{\circledast}$ FI27 [BASF Chem Trade GmbH, Burgbernheim, Germany]) and water phase on polymeric micelle mean size (A) and size distribution (B). Polymeric micelles were produced by the microfluidic device depicted in Figure I (microfluidics) (filled bars and circles) and, for comparison, by conventional bulk mixing (conventional) (open bars and circles) at the reported R values. Pluronic FI 27 concentration was kept constant at $7.5 \mathrm{mM}$ for all experiments.

Note: Data represent the average of three independent experiments (run in triplicate) \pm standard deviation.

Abbreviations: DMSO, dimethyl sulfoxide; PM, polymeric micelle; R, volumetric ratio of organic solution to water.

data referring to PMs produced by conventional bulk mixing procedure (using the same chemical composition) are also displayed. Figure 2 shows there is significant difference in the relationship between PM mean size and R exhibited by the two production methods. In the microfluidic system, PM size increases approximately linearly with an $\mathrm{R}$ increase, while on the contrary no clear correlation can be found for the bulk procedure. PMs produced by microfluidics display a smaller mean diameter $(52-61 \mathrm{~nm})$ in comparison to those by conventional bulk mixing $(65-74 \mathrm{~nm})$. In addition, the 
use of microfluidic reactors resulted in the production of PMs with significantly lower polydispersity (Figure 2B). The superior dimensional characteristics of PMs obtained by microfluidics were attributed to the controlled removal of DMSO in a precise fashion. This feature was feasible thanks to the fast and adjustable mixing process within microfluidic channels. In contrast, PMs prepared by the conventional procedure present a much broader dimensional distribution as demonstrated by the thick tail present in the frequency distribution curve (Figure 2B).

Further evidence of the superior controllability and reproducibility of microfluidics is demonstrated by the smaller standard deviation of the mean radius in PMs produced by microfluidics as compared with those produced by conventional procedure (Figure 2A). In addition, the results from different runs in the microfluidic system showed only a small variation in size $( \pm 3 \mathrm{~nm}$ compared to $\pm 11 \mathrm{~nm}$ from bulk systems), demonstrating the robustness of microfluidic production in terms of controllability and reproducibility.

\section{Effects of polymer concentration on PM characteristics}

The initial polymer concentration represents another important parameter to be considered especially for drug delivery applications. It is well know that PM in vivo stability is related to both polymer concentration and critical micellization concentration. In this respect, it should be considered that PMs after intravenous administration undergo dilution by circulating blood, usually about 25 -fold, in the case of a bolus injection. ${ }^{39}$

Therefore, the effects of polymer concentration and hydrodynamic flow focusing conditions on PM size were examined. Polymer concentrations were selected in order to be sufficiently high to prevent, after administration, a possible premature disassembly of PMs and the consequent release of the contained drug. An initial polymer concentration ranging between 7.5-15.0 $\mathrm{mM}$ was employed, giving a final concentration of 1.0-2.0 $\mathrm{mM}$ (at $\mathrm{R}=0.13$ ); notably, these values are about 15-30 times higher than Pluronic critical micellization concentration $(69 \mu \mathrm{M}) .{ }^{45}$

Different polymer concentrations resulted in PMs with a similar dimensional trend that corresponded with increasing R; only slightly larger dimensions occurred for PMs produced at high polymer concentration $(15.0 \mathrm{mM})$. This small but appreciable effect has been tentatively attributed to the viscosity increase of the polymeric solution, which increased from $9.1 \cdot 10^{-3}$ to $28.2 \cdot 10^{-3} \mathrm{~Pa} \cdot$ second. As a result, the mixing process was consistently slowed down, due to a smaller diffusion coefficient; this effect allowed the unimer aggregation to take place for a longer time, resulting in slightly larger PMs. ${ }^{44}$ In contrast, no significant differences were observed for the size distributions of PMs produced at low or high polymer concentrations (Figure 3B-D).

\section{Effects of MTH encapsulation on PM characteristics}

Figure 4 reports the dimensions of PMs produced by microfluidics or bulk mixing in the presence of different concentrations of MTH: $10.0 \mu \mathrm{M}, 32.5 \mu \mathrm{M}$, and 55.0 $\mu \mathrm{M}$; $\mathrm{R}=0.03$. Pluronic F127 concentration was kept constant at $15.0 \mathrm{mM}$ for all the experiments. Both preparation procedures resulted in the formation of PMs larger in size with respect to their empty counterparts. This result could be explained by the intercalation of MTH molecules (possessing amphiphilic properties) within the polymer chains into the core/shell interfacial region of the micelles. ${ }^{46}$ The presence of "guest" drug molecules is likely to decrease on one side of the interactions between unimers during the PM aggregation process ${ }^{40}$ and on the other side to increase interfacial surface area. ${ }^{47}$ However, how MTH and, in general, a nonprecipitating amphiphilic molecule interferes with the PM's precipitation process is beyond the scope of this manuscript and remains the subject of future research.

At concentration of $55.0 \mu \mathrm{M}, \mathrm{MTH}$ caused an increase in PM mean radius from $56 \mathrm{~nm}$ to $61 \mathrm{~nm}$ and from $75 \mathrm{~nm}$ to $98 \mathrm{~nm}$ for microfluidic and conventional procedures, respectively (Figure 4). As is clearly evident, MTH had a notable effect especially during the bulk mixing procedure, where it caused the formation of a thick tail of PMs with a characteristic radius above $120 \mathrm{~nm}$. In contrast, it caused only a small increase in the size of PM-MTH produced by microfluidics whereas the three MTH concentrations, that employed more than $90 \%$ of the produced PMs, had a characteristic radius below $110 \mathrm{~nm}$. Furthermore, the lower interbatch standard deviation found for microfluidic samples indicated higher reproducibility of the method (2.5-5.5 nm and 6.0-20.0 nm for microfluidic and conventional procedures, respectively).

The determination of drug association showed that MTH is preferentially partitioned in the micellar phase. Specifically, determinations in the present study demonstrated that about $85 \%$ and $83 \%$ (weight/weight) of the total MTH is associated to the micellar phase for microfluidics and bulk mixing preparation procedures, respectively. This result 


\section{A}

Pluronic F127 concentration (mM)

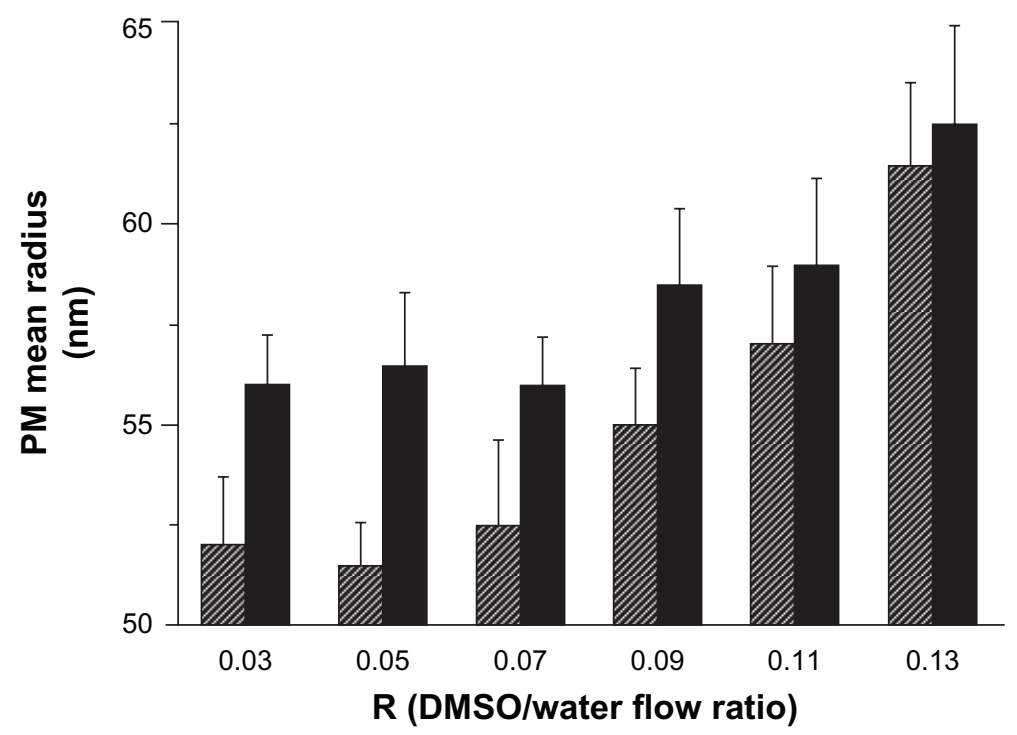

7.5

15.0

Pluronic F127 concentration (mM)

$7.5-\bigcirc$

15.0
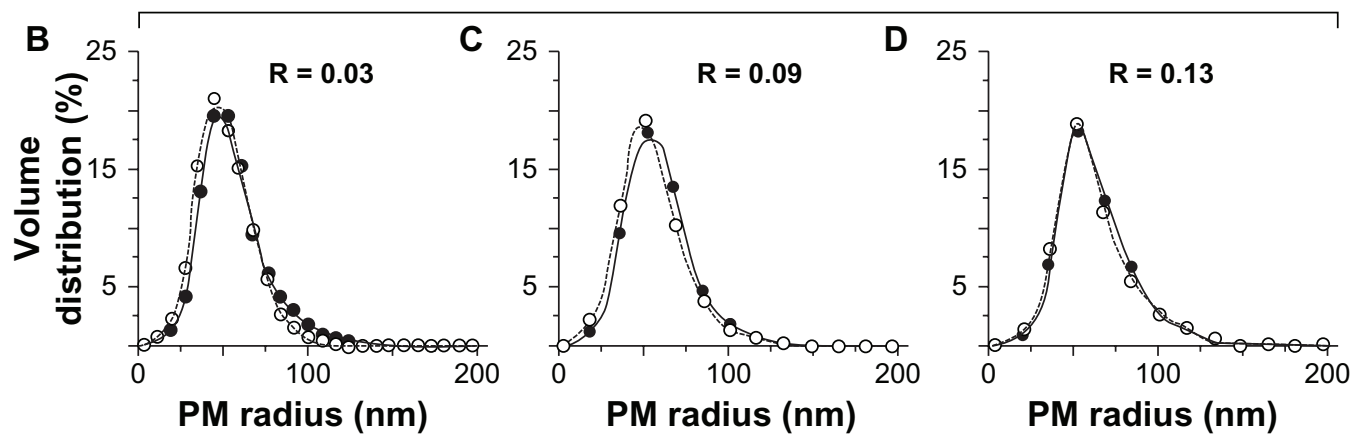

Figure 3 Effect of Pluronic ${ }^{\circledR}$ FI 27 (BASF Chem Trade GmbH, Burgbernheim, Germany) concentration and R on polymeric micelle mean size (A) and size distribution (B-D). Polymeric micelles were produced by microfluidics, at the reported R values, using a $7.5 \mathrm{mM}$ (striped bar, open circles) and I5.0 mM (filled bars and circles) Pluronic FI27 solution.

Note: Data represent the average of three independent experiments (run in triplicate) \pm standard deviation.

Abbreviations: DMSO, dimethyl sulfoxide; PM, polymeric micelle; R, volumetric ratio of organic solution to water.

is supported by the $\operatorname{drug} \log P(1.290 \pm 1.454)$, indicating the slight lipophilic nature of MTH molecule. ${ }^{26}$

\section{Optimization of PM-MTH production by DoE approach}

In order to comparatively study the influence of experimental parameters on PM characteristics produced by microfluidic versus conventional bulk mixing, a statistical data analysis based on a DoE approach was performed.

The DoE approach used was a full factorial design plus two center points. It studies the effects of design variables independently from each other, including interaction terms.
Full factorial designs include enough experiments to allow use of a model with all interactions. Thus, they are a logical choice if one intends to study interactions in addition to main effects.

From the initial COST results (Figures 2-4), previous studies, and as already discussed, two factors were included in the DoE approach to study their effect on the characteristics of PMs, namely R and drug concentration. . $^{30,44}$

The response variables and their target ranges were selected, taking into consideration that it is well-established that size and size distribution are major factors determining the pharmacokinetic and biodistribution of PMs in vivo. ${ }^{20,31,48}$ 

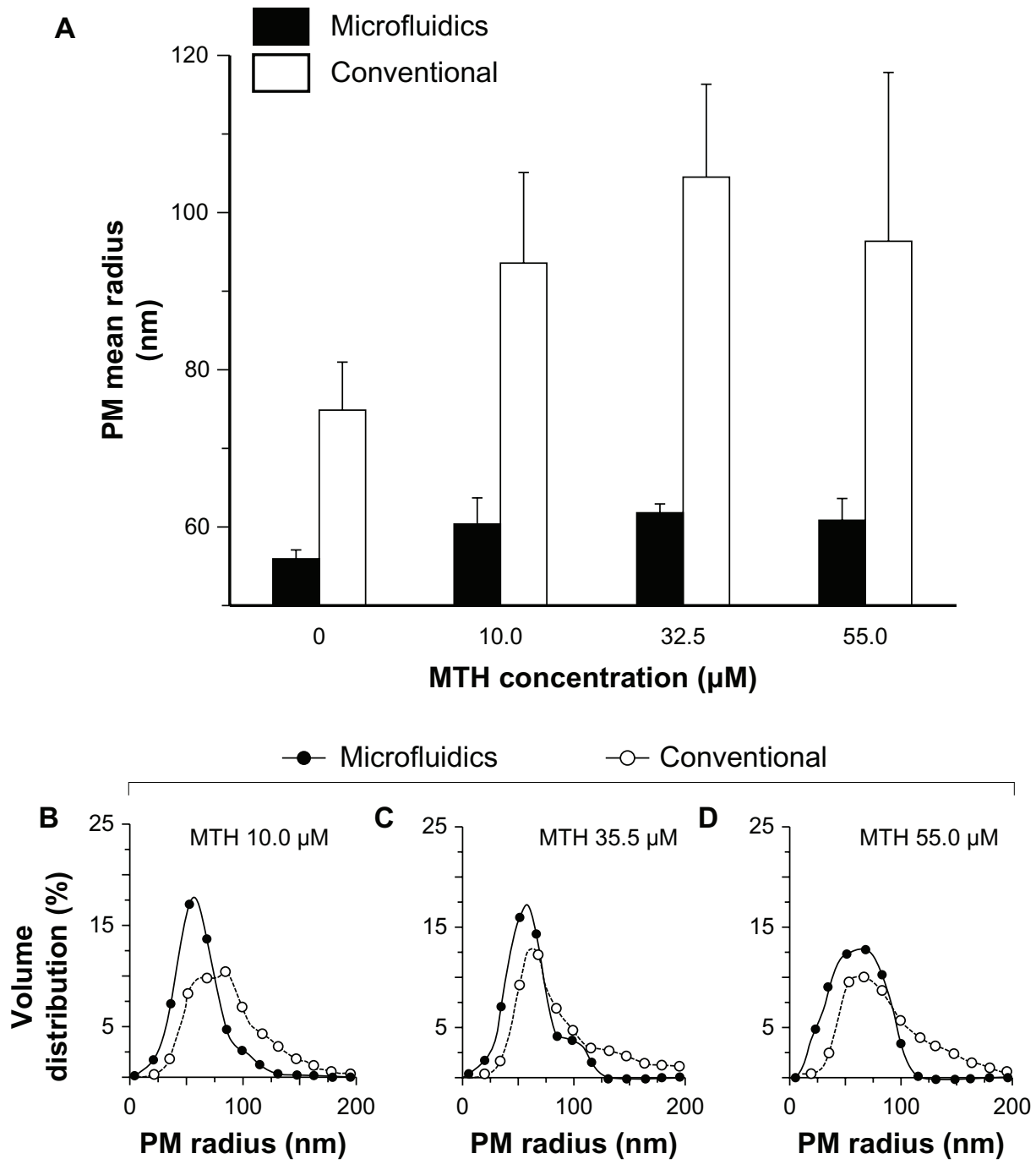

Figure 4 Effect of preparation procedure and mithramycin concentration on mean size (A) and size distribution (B-D) of mithramycin encapsulated in polymeric micelles. Pluronic ${ }^{\circledR}$ FI27 (BASF Chem Trade GmbH, Burgbernheim, Germany) concentration was kept constant at I5.0 mM. Mithramycin encapsulated in polymeric micelles was prepared by microfluidics (filled bars) or conventional bulk mixing (conventional) (open bars). Size distribution of polymeric micelles produced with $10.0 \mu \mathrm{M}$ (B), $32.5 \mu \mathrm{M}$ (C), and $55.0 \mu \mathrm{M}$ (D) mithramycin solution by microfluidics (filled circles) or bulk mixing (open circles) are reported.

Note: Data represent the average of three independent experiments (run in triplicate) \pm standard deviation.

Abbreviations: MTH, mithramycin; PM, polymeric micelle.

Typically, PMs with mean radius 50-100 $\mathrm{nm}$ maximize stealth properties, reducing recognition by immune system components with prolonged plasma circulation times, as compared to those with larger diameter. ${ }^{16,17,21,49,50}$ In addition, PMs larger than $200 \mathrm{~nm}$ in diameter have been demonstrated to be removed from circulation by spleenic filtration at interendothelial cell slits. ${ }^{50-53}$ Taking into account the above reported consideration, the goal was to obtain PMs with sizes $50-100 \mathrm{~nm}$ in radius, a size distribution as narrow as possible, and a high $\operatorname{drug}_{200}$. Particularly, the response factor $\operatorname{drug}_{200}$ was taken into consideration to estimate the amount of drug associated in PMs larger than $200 \mathrm{~nm}$ in diameter.

The experiments were designed to simultaneously investigate the effects of the factors ( $\mathrm{R}$ and concentration) on the responses (size, standard deviation, and $\operatorname{drug}_{200}$ ) (see explanations in Table 1). Each factor was tested at three levels and two additional center points were added, in order to get an estimation of the experimental error. In Table 2 and Figure 5 the complete list of experimental data and results are reported.

The mathematical equation employed for the $3^{2}$ full factorial design was:

$$
\mathrm{y}=\mathrm{A}_{0}+\mathrm{A}_{1} \text { concentration }+\mathrm{A}_{2} \mathrm{R}+\mathrm{A}_{3} \text { concentration } \cdot \mathrm{R}
$$

where $\mathrm{A}_{\mathrm{o}}$ represents the global mean and $\mathrm{A}_{1}$ represents the other regression coefficients.

Three-dimensional graphs (Figure 5) show the factor influence on size, standard deviation, and $\operatorname{drug}_{200}$ of 
Table I Design of experiments study of the influence of experimental parameters on the PM characteristics: investigated factors and responses

\begin{tabular}{|c|c|c|c|}
\hline Parameter & Abbreviation & Meaning & Range \\
\hline \multicolumn{4}{|l|}{ Factor } \\
\hline Volumetric ratio & $\mathrm{R}$ & $\begin{array}{l}\text { The volumetric ratio between the polymeric solution in DMSO } \\
\text { and water phase }\end{array}$ & $0.03-0.13$ \\
\hline Mithramycin concentration & Conc & $\begin{array}{l}\text { The concentration of the MTH solution in DMSO, employed } \\
\text { for the PM production }\end{array}$ & $10.0-55.0 \mu \mathrm{M}$ \\
\hline \multicolumn{4}{|l|}{ Responses } \\
\hline Polymeric micelle size & Size & $\begin{array}{l}\text { The mean diameter of polymeric micelle containing MTH as } \\
\text { determined by dynamic light scattering }\end{array}$ & \\
\hline $\begin{array}{l}\text { Standard deviation of polymeric } \\
\text { micelle size }\end{array}$ & SD & $\begin{array}{l}\text { The standard deviation of the mean diameter of polymeric } \\
\text { micelle containing MTH }\end{array}$ & \\
\hline $\begin{array}{l}\text { Mithramycin in polymeric } \\
\text { micelles }<200 \mathrm{~nm}\end{array}$ & Drug $_{200}$ & $\begin{array}{l}\text { The percentage of drug encapsulated in polymeric micelles } \\
\text { with a mean diameter } \leq 200 \mathrm{~nm}\end{array}$ & \\
\hline
\end{tabular}

Abbreviations: MTH, mithramycin; DMSO, dimethyl sulfoxide; PM, polymeric micelle.

PMs produced by microfluidics (red surfaces) versus conventional bulk mixing (green surfaces). The reported results (see also Table 2) indicate the superior overall quality of PMs produced by microfluidics. Indeed, the red surfaces show a better quality of the produced PMs in terms of size, standard deviation, and $\operatorname{drug}_{200}$. Specifically, for the microfluidic protocol, a clear trend of the factor effects on the size characteristics has been found: an increase in the factor $\mathrm{R}$ from low to high level (0.03-0.13) resulted in a moderate increase in the response size that rose from about $60 \mathrm{~nm}$ to around $90 \mathrm{~nm}$, while the response standard deviation remained almost constant, with only a slight increase as a result of increased $\mathrm{R}$ and concentration.

These results clearly demonstrated the precise control over the mixing under laminar flow conditions, resulting in predictable continuous flow preparation of PMs of controlled size (Figure 5A and B). In contrast, the bulk mixing led to a lack of a dimensional correlation between experimental parameters and PM size; it is indeed evident that the bulk mixing caused the formation of large and polydisperse PMs. The limited size control is likely due to the chaotic nature of mixing under the turbulent conditions associated with bulk mixing procedures.

The analysis of drug ${ }_{200}$ gives a coarse indication of the percentage of drug that is associated in small PMs, which can be taken up by the cells through nonphagocytic translocation. In this respect, it has been shown that a reduction of size was a critical factor to improve such mechanism of translocation within blood cells. ${ }^{32}$ The concentration factor deeply influences this response; specifically, an increase of concentration was accompanied by a decrease of drug ${ }_{200}$ (Figure 5C and Table 2) for PM-MTH produced with bulk mixing. In contrast, the microfluidic-based procedure is much less affected by the loss of drug during the filtration step, as evident by the higher value for $\operatorname{drug}_{200}$. As a general explanation of the drug $_{200}$ trend, the progressive rise of MTH molecules probably causes the formation of an increasing number of large PM-MTH aggregates. Interestingly, at the same concentration level, the micelles produced by bulk mixing at different $\mathrm{R}$ show a similar drug $_{200}$, indicating that $\mathrm{R}$ has only a minor effect on $\operatorname{drug}_{200}$ for this preparation procedure.

Once again, the microfluidic procedure shows superior control over the final micelle characteristics; for instance, the highest drug $_{200}$ is achieved at an intermediate $\mathrm{R}$ value $(0.08)$. The authors believe that this effect could be related to the complex interactions between molecular and hydrodynamic phenomena occurring during the micelle assembly, even if, at this stage, there is no sufficient experimental evidence to corroborate this hypothesis.

The DoE analysis also offers indications for the development of a robust preparation procedure that is of primary importance for the therapeutic application of PMs, where an efficient control of the PMs' characteristics is required to achieve an optimal and reproducible therapeutic efficacy. In this respect, the validity and significance of the DoE model was assessed by analysis of variance. Table 3 reports the percentage of the variation of response explained by the model $\left(\mathrm{R}^{2}\right)$ and the model reproducibility for both bulk and microfluidic mixing procedures. The reported results demonstrate that all the obtained experimental data fit well with the model, indicating good reproducibility. Moreover, the high values of both coefficients indicate that the model has good validity $\left(\mathrm{R}^{2}\right)$ and significant reproducibility, especially in the case of PMs produced by microfluidics. 
Table 2 Experimental design matrix and results of the design of experiments approach for the preparation of mithramycin based on polymeric micelles

\begin{tabular}{|c|c|c|c|c|c|}
\hline \multirow[t]{2}{*}{ Run } & \multicolumn{2}{|l|}{ Factors } & \multicolumn{3}{|c|}{ Responses } \\
\hline & Concentration $^{a}$ & $\mathbf{R}^{\mathbf{b}}$ & Size $^{c}$ & $\begin{array}{l}\text { Standard } \\
\text { deviation }\end{array}$ & $\operatorname{Drug}_{200}{ }^{e}$ \\
\hline \multicolumn{6}{|c|}{ Microfluidics } \\
\hline \#I & 10.0 & 0.3 & 60.0 & 21.0 & 77.0 \\
\hline \#2 & 55.0 & 0.3 & 61.0 & 22.0 & 58.6 \\
\hline \#3 & 10.0 & 0.13 & 81.0 & 23.0 & 92.3 \\
\hline$\# 4$ & 55.0 & 0.13 & 90.0 & 28.0 & 48.1 \\
\hline \#5 & 10.0 & 0.08 & 69.0 & 21.0 & 89.8 \\
\hline$\# 6$ & 55.0 & 0.08 & 71.0 & 23.0 & 55.3 \\
\hline$\# 7$ & 32.5 & 0.03 & 62.0 & 22.0 & 84.7 \\
\hline$\# 8$ & 32.5 & 0.13 & 73.0 & 24.0 & 65.0 \\
\hline \#9 & 32.5 & 0.08 & 76.0 & 25.0 & 86.8 \\
\hline$\# 10$ & 32.5 & 0.08 & 74.0 & 21.0 & 86.4 \\
\hline \#II & 32.5 & 0.08 & 75.0 & 26.0 & 87.0 \\
\hline \multicolumn{6}{|c|}{ Bulk mixing } \\
\hline$\# \mathrm{lb}$ & 10.0 & 0.3 & 94.0 & 47.0 & 72.3 \\
\hline$\# 2 b$ & 55.0 & 0.3 & 97.0 & 56.0 & 35.0 \\
\hline \#3b & 10.0 & 0.13 & 92.0 & 36.0 & 79.7 \\
\hline$\# 4 \mathrm{~b}$ & 55.0 & 0.13 & 106.0 & 37.0 & 35.8 \\
\hline$\# 5 b$ & 10.0 & 0.08 & 109.0 & 67.0 & 76.1 \\
\hline \#6b & 55.0 & 0.08 & 100.0 & 52.0 & 49.2 \\
\hline \#7b & 32.5 & 0.03 & 105.0 & 70.0 & 59.1 \\
\hline$\# 8 \mathrm{~b}$ & 32.5 & 0.13 & 123.0 & 52.0 & 49.2 \\
\hline$\# 9 b$ & 32.5 & 0.08 & 120.0 & 73.0 & 38.7 \\
\hline$\# 10 b$ & 32.5 & 0.08 & 106.0 & 55.0 & 34.9 \\
\hline$\#|l| b$ & 32.5 & 0.08 & 110.0 & 49.0 & 37.5 \\
\hline
\end{tabular}

Notes: ${ }^{a}$ Concentration $(\mu \mathrm{M})$ of the mithramycin solution in dimethyl sulfoxide employed for production of polymeric micelles; bvolumetric ratio between the polymeric solution in dimethyl sulfoxide and water phase; ' $m e a n$ radius of the obtained polymeric micelles as determined by dynamic light scattering $(\mathrm{nm})$; ${ }^{\mathrm{d}}$ standard deviation of the mean radius measurements ( $n=5$, each measured in quintuplicate); epercentage of drug associated in polymeric micelles with diameter $\leq 200 \mathrm{~nm}$.

\section{Effect of PM-MTH on proliferation and differentiation of $\mathrm{K} 562$}

After DoE analysis, the optimal formulation $(\mathrm{R}=0.03$ and concentration $=32.5 \mu \mathrm{M}$ ) was selected due to the small size and dimensional homogeneity of the produced PM-MTH. Such formulation was tested in vitro to investigate its biological activity and effectiveness as potential treatment for $\beta$-thalassemia. For comparison, PM-MTH produced both by conventional bulk mixing and microfluidic procedures were considered.

K562 cells, derived from a patient with chronic myeloid leukemia in terminal blast cell crisis, exhibit erythroid features and can be induced to produce embryonic and fetal, but not adult, hemoglobin. Therefore, such a cell line can be conveniently employed as model for studying hemoglobin production upon induction to differentiation. ${ }^{7}$ In this respect, it was initially determined, as reported in Figure 6, whether
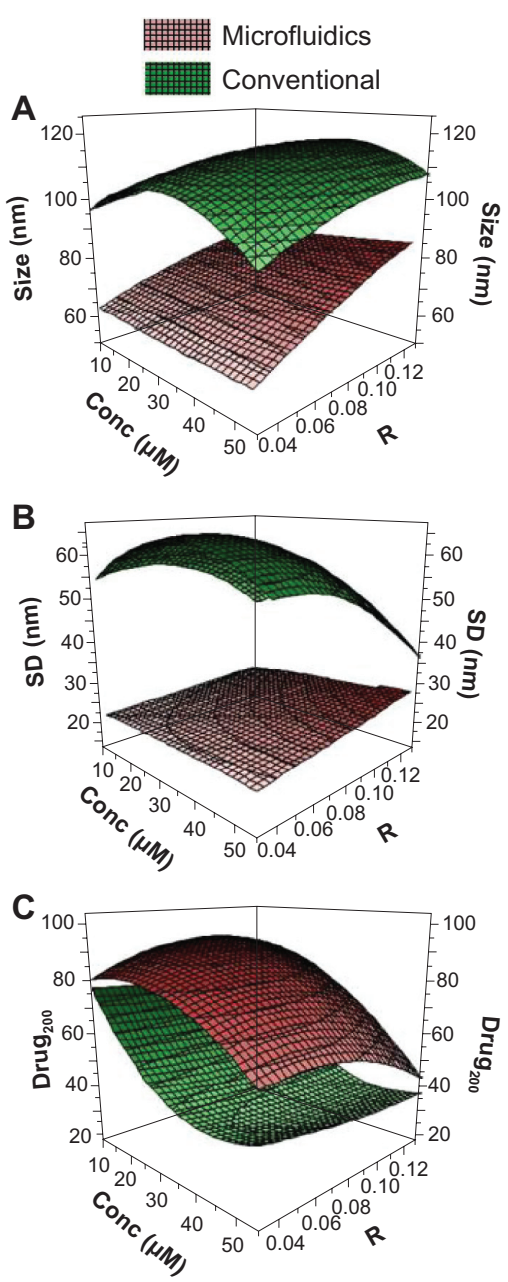

Figure 5 Design of experiments analysis of the preparation of mithramycin encapsulated in polymeric micelles. Response surface plots for mithramycin encapsulated in polymeric micelles obtained by microfluidics (red surfaces) or conventional bulk mixing (green surfaces). The following factors and responses were respectively investigated: concentration and $R$ versus size $(\mathbf{A})$, concentration and $R$ versus standard deviation (B), and concentration and $R$ versus drug $g_{200}(\mathbf{C})$.

Note: Size is the mean radius of the obtained polymeric micelles as determined by dynamic light scattering.

Abbreviations: conc, concentration of the mithramycin solution in dimethyl sulfoxide employed for production of polymeric micelles; drug $_{200}$, percentage of drug associated in polymeric micelles with diameter $\leq 200 \mathrm{~nm}$; R, volumetric ratio between the polymeric solution in dimethyl sulfoxide and water phase; SD, standard deviation of the mean radius measurements $(n=5$, each measured in quintuplicate).

Table 3 Analysis of variance of the model

\begin{tabular}{llll}
\hline Responses & N & $\mathbf{R}^{2}$ & Reproducibility \\
\hline Direct mixing & & & \\
Size & II & 0.632 & 0.445 \\
Standard deviation & II & 0.576 & -0.445 \\
Drug $_{200}$ & II & 0.845 & 0.987 \\
Microfluidic mixing $_{\text {Size }}$ & & \\
Standard deviation & II & 0.83 I & 0.992 \\
Drug $_{200}$ & II & 0.646 & 0.098 \\
\hline
\end{tabular}

Abbreviations: Drug $_{200}$, percentage of drug associated in polymeric micelles with diameter $\leq 200 \mathrm{~nm} ; \mathrm{N}$, number of experiments; $R^{2}$, percentage of the variation of the response explained by the model. 


\section{Free MTH}

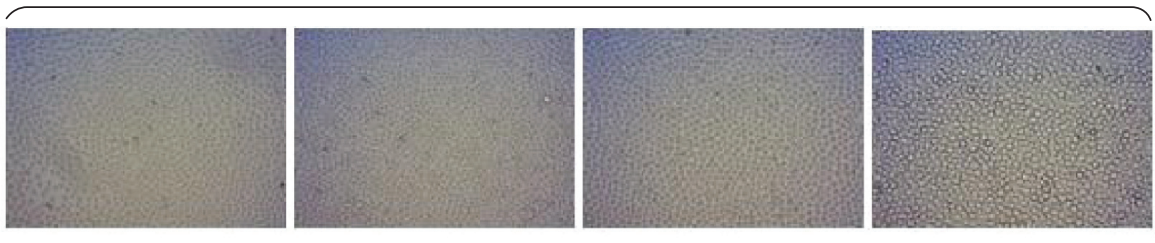

Microfluidics

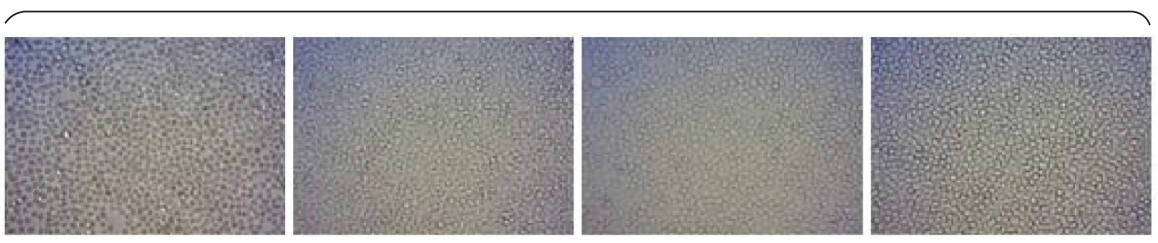

Conventional

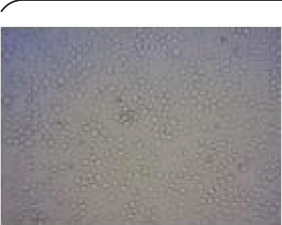

100.0

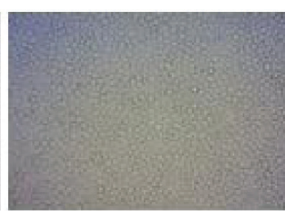

50.0

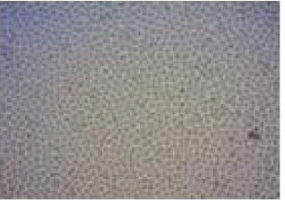

25.0

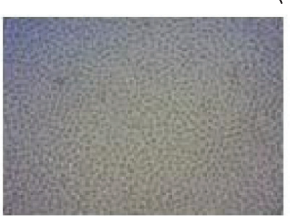

$12.5 \mathrm{nM}$
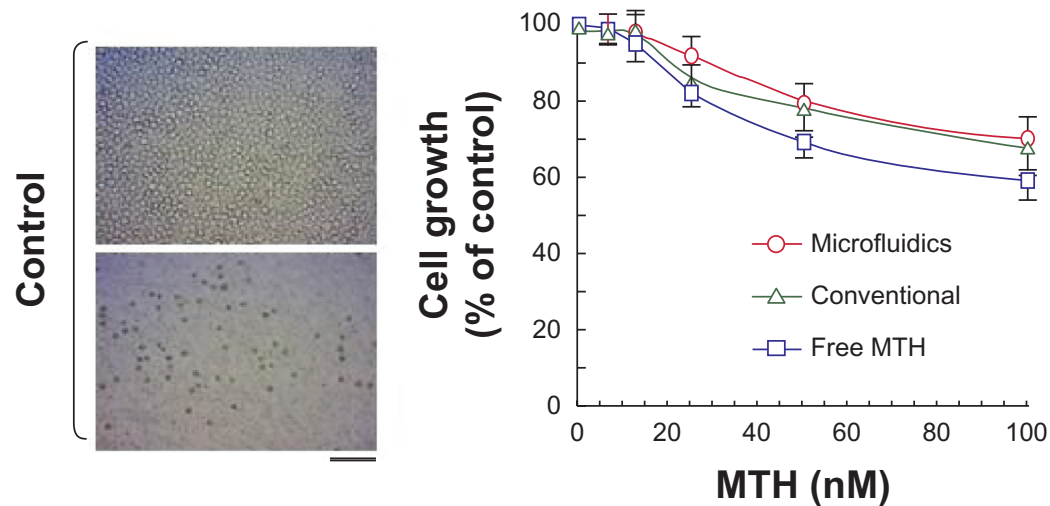

Figure 6 Effect of mithramycin on growth of K562 cells. Microphotographs showing K562 cell density after 3 days of culture in the absence (control) or presence of the indicated mithramycin concentrations (nM). Corresponding graph: free mithramycin (squares), mithramycin encapsulated in polymeric micelles prepared by microfluidics (circles), or conventional bulk mixing (triangles).

Notes: Data represent the average of three independent experiments (run in triplicate) \pm standard deviation. Bar corresponds to $100 \mu \mathrm{m}$. Abbreviation: MTH, mithramycin.

PM-MTH could affect the proliferation of K562 cells when employed at the concentrations exhibiting effects on differentiation. For comparison, Figure 6 reports the data obtained by the free drug. Cells were cultured for 3 days in the presence of the indicated concentrations of MTH, then added to the culture medium in form of free drug or encapsulated into PMs, and prepared by microfluidics or bulk mixing procedures. As shown by the proliferation versus MTH concentration graph, when the drug is employed in the range of concentrations comprised between $6-50 \mathrm{nM}$, it had only a limited effect on cell proliferation $(<35 \%)$. K562 cells cultured in the presence of Pluronic F127-empty
PMs (ie, without drug), prepared with the same chemical composition and using an identical experimental setup used for the production PM-MTH, displayed a viability and proliferation rate superimposable to the untreated control cells (data not shown).

Notably, the delivery by PM-MTH appears to have a positive effect on the cytotoxic activity of the drug. In fact PM-MTH for all the tested concentrations presented a slightly less pronounced effect on cell growth, with respect to the free drug. After having demonstrated that free MTH and PM-MTH, at concentrations below $50 \mathrm{nM}$, presented no significant inhibitory activity on cell growth, the ability 
A

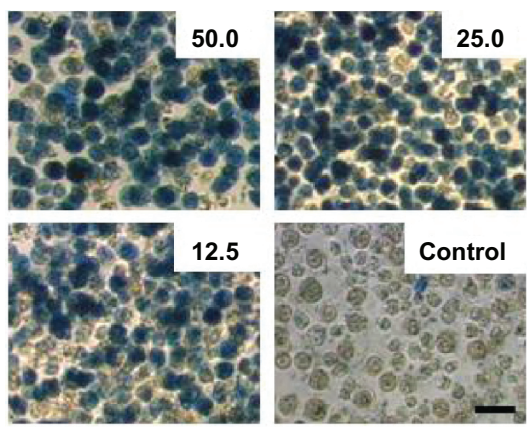

B

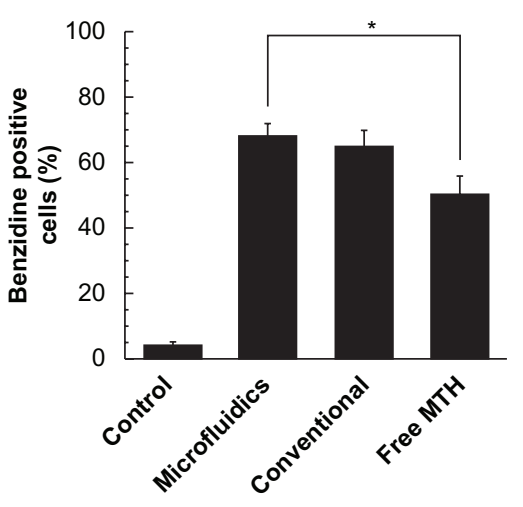

C

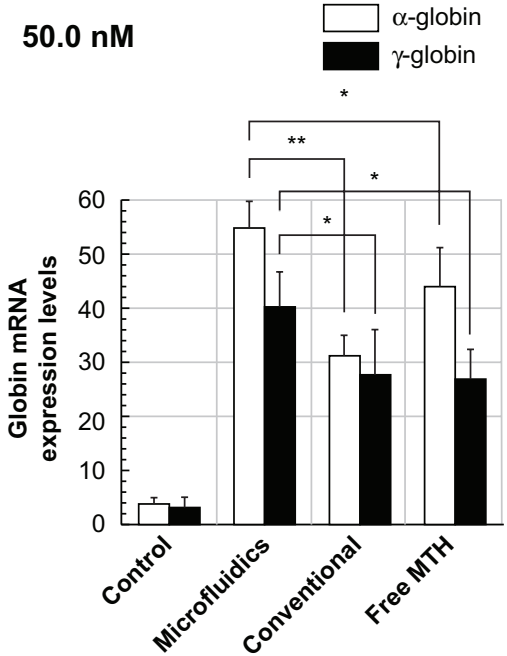

Figure 7 Effect of mithramycin on erythroid differentiation of $\mathrm{K} 562$ cells (A and B). Cells were cultured for 7 days in the presence of the indicated mithramycin concentrations $(n M)$, then added to the culture medium in form of free drug or associated in polymeric micelles, and prepared by microfluidics or conventional bulk mixing. (A) Microphotographs were taken after benzidine staining of cells treated with mithramycin encapsulated in polymeric micelles (microfluidics); thereafter cells were counted and the percent of benzidine-positive cells was determined and plotted. Scale bar $25 \mu \mathrm{m}$. (B) Data represent the average of three independent experiments (run in triplicate) \pm standard deviation (microfluidics versus free mithramycin $P<0.05$ ). (C) Effects of mithramycin on expression of globin genes. Cells were cultured for 4 days in the presence of mithramycin $(50 \mathrm{nM})$, then added to the culture medium in form of free drug or associated in polymeric micelles, and prepared by microfluidics or conventional bulk mixing. Reverse transcriptasepolymerase chain reaction was performed for quantification of $\alpha$-globin (open bars) and $\gamma$-globin (filled bars) messenger ribonucleic acid sequences.

Notes: Data represent fold content in respect to control uninduced cells (average \pm standard deviation of three independent experiments); $\alpha$-globin: microfluidics versus conventional $P<0.00 \mathrm{I}$, microfluidics versus free mithramycin $P<0.05 ; \gamma$-globin: microfluidics versus conventional $P<0.05$, microfluidics versus free mithramycin $P<0.05$.

Abbreviations: MTH, mithramycin; mRNA, messenger ribonucleic acid. of PM-MTH to induce the erythroid differentiation of K562 cells was tested. Figure 7 shows that cell treatment at the indicated concentration of free MTH and PM-MTH induced a clearly evident erythroid differentiation, as proved by the intense positive blue color developed by the cells after benzidine staining, which indicates hemoglobin production (Figure 7A and B). Empty Pluronic F127 micelles, used as control, did not show any appreciable difference in cell differentiation when compared with untreated cells (data not shown). Notably, the delivery by micelles produced by microfluidics appears to have a positive effect on the differentiative activity of MTH, In fact, at 50.0 nM PM-MTH presented a slightly more pronounced differentiation, with respect to free drug (microfluidics versus free MTH $P<0.05$ ). However, benzidine staining indicates whether a single cell is undergoing erythroid differentiation and hemoglobin production, it does not give information on the levels of induction, in terms of accumulation of globin mRNA and hemoglobin within single benzidine-positive cell populations.

In order to obtain quantitative results and additional information at the molecular level, RT-PCR was performed, for the analysis of $\alpha$ - and $\gamma$-globin mRNA expression levels (Figure 7C). The quantification of $\beta$-globin mRNA was not performed, since it is known that the $\beta$-globin gene is not efficiently transcribed in K562 cells. ${ }^{54}$ The obtained results indicate that microfluidic-prepared PM-MTH are more active in inducing $\alpha$ - and $\gamma$-globin mRNA accumulation when compared to free MTH or PM-MTH prepared by bulk mixing ( $\alpha$-globin: microfluidics versus bulk $P<0.001$, microfluidics versus free MTH $P<0.05 ; \gamma$-globin: microfluidics versus bulk $P<0.05$, microfluidics versus free MTH $P<0.05$ ).

Smaller dimensions of the microfluidic-produced PMMTH, as compared with those produced by bulk procedure (Figure 5C and Table 2), might be the reason for more efficient cell internalization through nonphagocytic translocation mechanism, resulting in higher intracellular MTH concentration and relatively higher differentiative activity. Further investigations are underway in the authors' laboratory to elucidate the mechanism of this effect.

\section{Effect of PM-MTH on ErPCs}

As further investigation, it was analyzed whether MTH delivered by microfluidic-produced PM-MTH are able to stimulate $\mathrm{HbF}$ production in erythroid progenitor cells from $\beta$-thalassemia patients. ErPC were stimulated with erythropoietin in the absence of MTH (uninduced ErPC) or in the presence of MTH (either free drug or PM-MTH), 
A

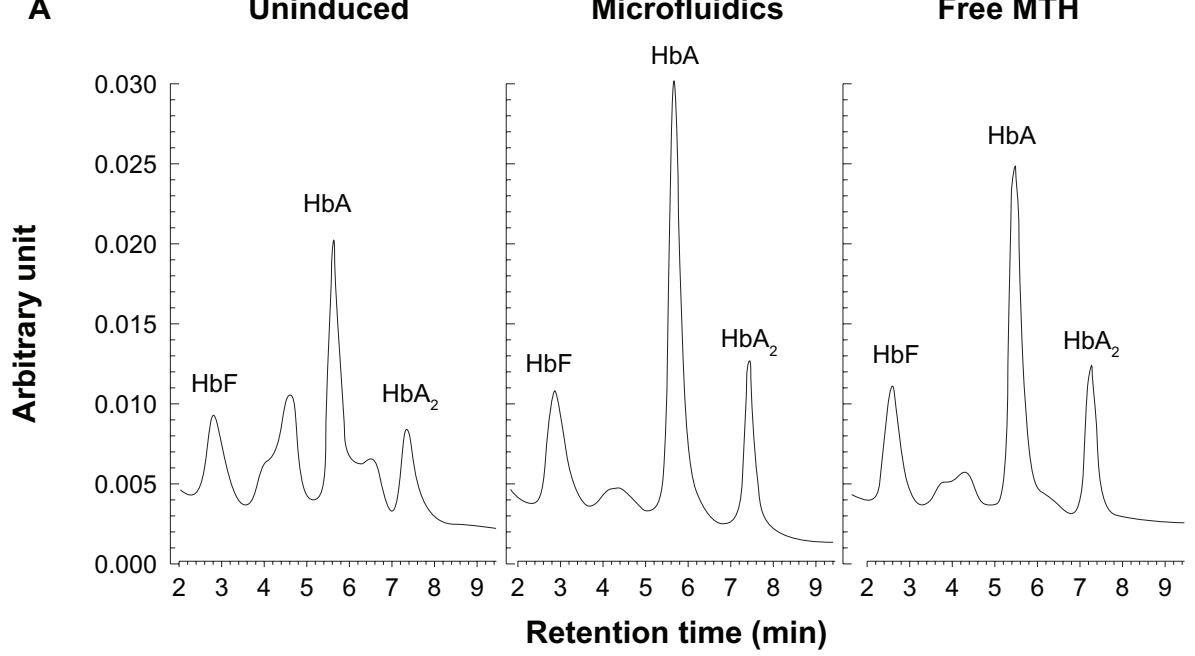

B

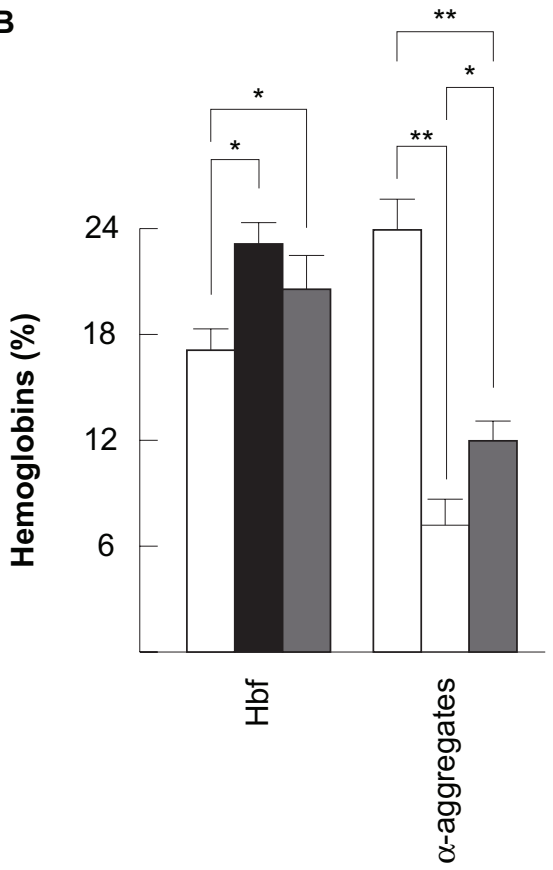

C
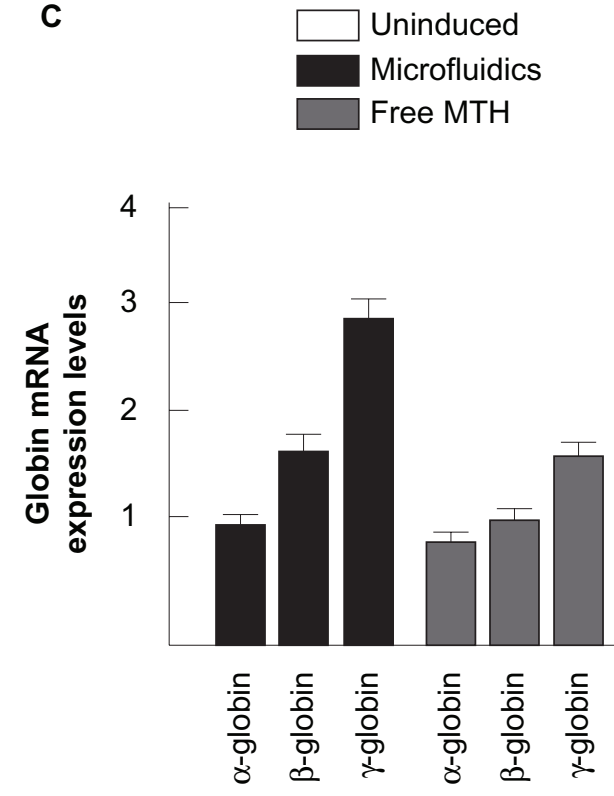

Figure 8 Effect of mithramycin encapsulated in polymeric micelles prepared by microfluidics on fetal hemoglobin production by erythroid precursor cells from a $\beta$-thalassemia patient. Erythroid precursor cells were cultured in phase II with only erythropoietin, or erythropoietin plus free mithramycin, or mithramycin encapsulated in polymeric micelles prepared by microfluidics. (A) High performance liquid chromatography profile of uninduced erythroid precursor cells (left), erythroid precursor cells induced by mithramycin encapsulated in polymeric micelles (center), and free mithramycin (right). (B) Quantitative effects on fetal hemoglobin and the production of $\alpha$-aggregates in erythroid precursor cells induced by mithramycin encapsulated in polymeric micelles (filled bars) and free mithramycin (grey bars). For comparison, data for uninduced erythroid precursor cells (open bars) are also reported (fetal hemoglobin: uninduced versus microfluidics $P<0.05$, uninduced versus free mithramycin $P<0.05 ; \alpha$-aggregates: uninduced versus microfluidics $P<0.00 \mathrm{I}$, uninduced versus free mithramycin $P<0.00 \mathrm{I}$, microfluidics versus free mithramycin $P<0.05$ ). (C) Reverse transcriptasepolymerase chain reaction analysis of erythroid precursor cells expressed as fold induction of $\alpha$-globin, $\beta$-globin, and $\gamma$-globin messenger ribonucleic acid production. Cells were treated with mithramycin encapsulated in polymeric micelles (black bar) or free drug (grey bars).

Note: Data represent the average of three independent determinations \pm standard deviation.

Abbreviations: HbA, adult hemoglobin; HbF, fetal hemoglobin; min, minutes; MTH, mithramycin; mRNA, messenger ribonucleic acid.

and the lysates were analyzed by HPLC. As demonstrated by Figure 8, an increase of $\mathrm{HbF}$ is observed from $17.23 \%$ (uninduced ErPC) to $22.71 \%$ (ErPC treated with PM-MTH). As expected, free MTH stimulates an $\mathrm{HbF}$ increase (20.35\%) but to a lower extent. Of interest is the parallel reduction of $\alpha$-globin aggregates which represent $24.39 \%$ of the total hemoglobin (for uninduced ErPC), both in ErPC treated with PM-MTH (6.71\%) or free MTH (11.30\%) (HbF: uninduced versus microfluidics $P<0.05$, uninduced versus free MTH $P<0.05$; $\alpha$-aggregates: uninduced versus microfluidics $P<0.001$, uninduced versus free MTH $P<0.001$, microfluidics versus free MTH $P<0.05$ ). 
The decrease of $\alpha$-globin aggregates following treatment with $\mathrm{HbF}$ inducers is of a great importance, since the excess of $\alpha$-globin production by $\beta$-thalassemia patients is responsible for the clinical symptoms of this pathology. When the HPLC results are reported on a cell basis, $\mathrm{HbF}$ was $0.12 \pm 0.04$ in control cells, $0.24 \pm 0.06$ in PM-MTH-treated cells, and $0.20 \pm 0.06$ in ErPC treated with free MTH.

These findings are further sustained by RT-PCR analyses on mRNA samples (Figure 8C). No increase of $\alpha$-globin mRNA is observed in both ErPC treated with PM-MTH or free MTH, while PM-MTH is able to induce $\gamma$-globin mRNA (2.87-fold) and $\beta$-globin mRNA (1.68-fold) more efficiently than free MTH (1.64-fold and 1.14-fold, respectively).

\section{Conclusion}

A microfluidic-based protocol for the production of a micellar delivery system for MTH was developed and examined in comparison to micelles produced with conventional methods. The effects of different production parameters were investigated and their effects on the size and size distribution were statistically evaluated. The results indicate that MTH can be efficiently formulated as micellar nanoparticles in a controlled and reproducible manner not achievable by conventional bulk method. After preparation and characterization, PM-MTH prepared both by microfluidic and bulk methods were tested in vitro to investigate their biological activity and effectiveness as potential treatment for $\beta$-thalassemia. Interestingly, PM-MTH exhibited a slightly lower toxicity and more pronounced differentiative activity when compared to the free drug. Moreover, the presented data suggest that PM-MTH produced by microfluidics is able to induce, at high levels, globin mRNA accumulation in K562 cells. Of note, the RT-PCR data, obtained using ErPCs, demonstrated that PM-MTH, as the most effective $\mathrm{HbF}$ inducer, is able to induce $\beta$-globin and even more efficiently $\gamma$-globin gene expression without stimulating $\alpha$-globin gene expression. This result is sustained by the HPLC analyses, which showed an increase of $\mathrm{HbF}$ and a decrease of $\alpha$-globin aggregates in ErPC treated with erythropoietin in the presence of PM-MTH produced by microfluidics.

In conclusion, the current paper suggests that PM-MTH might be of great interest in inducing $\mathrm{HbF}$ in thalassemic patients, representing an important first step towards the future development of micellar and other nanomedicinerelated therapeutic protocols for $\beta$-thalassemia. Also, considering the simplicity and reproducibility of the microfluidic technology, the presented production strategy might be suitable for personalized medicine applications, which require controllable PM characteristics and consistency from batch to batch.

These results, representing an important first step in clinical development, should be considered with caution as a possible therapeutic treatment of $\beta$-thalassemia patients for at least two reasons. First, ErPC isolated from $\beta$-thalassemia patients exhibit very different starting levels of $\mathrm{HbF}$ and different response to $\mathrm{HbF}$ inducers, including MTH. ${ }^{9}$ Accordingly, the analysis of several patients with different (a) genotype, (b) starting levels of $\mathrm{HbF}$, and (c) response to hydroxyurea (the most frequently used $\mathrm{HbF}$ inducer in the therapy of patients affected by $\beta$-thalassemia and sickle-cell anemia) is required before proposing this strategy for $\beta$-thalassemia therapy. Second, with respect to the toxicity of MTH, two considerations should be made: (a) MTH analogs exhibiting safer pharmacological features and improved therapeutic index have been recently developed ${ }^{55,58}$ and (b) novel transgenic mouse systems are available to study the effects in vivo of either free MTH and PM-MTH formulations. ${ }^{59,60}$ For instance, the recently humanized transgenic mice carrying the entire human $\beta$-globin locus are very appealing for testing possible $\mathrm{HbF}$ inducers, since this experimental model does not suffer by the fact that an HbF-like hemoglobin is not produced by normal mice. ${ }^{9}$

A further consideration is related to possible applications of PM-MTH to other pathologies including neoplastic diseases. ${ }^{60}$ In this respect, it would be very interesting to verify the activity of PM-MTH on tumor cell lines and in vivo tumor cell systems.

Finally, the comparison of the effects of free MTH and PM-MTH on the expression of the genome should be evaluated; transcriptomic as well as proteomic analyses are now widely used to describe the effects of therapeutic molecules, including MTH, on the overall genome expression. Microarray analysis and proteomic studies will conclusively demonstrate whether or not PM-mediated delivery of MTH alters the effects on gene expression.

\section{Acknowledgments}

The authors would like to thank the United Kingdom Engineering and Physical Sciences Research Council (EPSRC) for supporting this study financially. BASF Chem Trade GmbH (Burgbernheim, Germany) is thanked for providing polymer Pluronic ${ }^{\circledR}$ F127. Dr Lefteris Danos of School of Engineering Sciences, University of Southampton, is thanked for the technical assistance. 


\section{Disclosure}

The authors report no conflicts of interest in this work.

\section{References}

1. Barcelo F, Ortiz-Lombardia M, Martorell M, et al. DNA-binding characteristics of mithramycin and chromomycin analogues obtained by combinatorial biosynthesis. Biochemistry. 2010;49(49): 10543-10552.

2. Koller CA, Miller DM. Preliminary observations on the therapy of the myeloid blast phase of chronic granulocytic leukemia with plicamycin and hydroxyurea. N Engl J Med. 1986;315(23):1433-1438.

3. Yuan P, Wang L, Wei D, et al. Therapeutic inhibition of Sp1 expression in growing tumors by mithramycin a correlates directly with potent antiangiogenic effects on human pancreatic cancer. Cancer. 2007;110(12):2682-2690.

4. Ryuto M, Ono M, Izumi $\mathrm{H}$, et al. Induction of vascular endothelial growth factor by tumor necrosis factor in human glioma cells. $J$ Biol Chem. 1996;271(45):28220-28228.

5. Campbell VW, Davin D, Thomas S, et al. The G-C specific DNA binding drug, mithramycin, selectively inhibits transcription of the $\mathrm{C}-\mathrm{MYC}$ and C-HA-RAS genes in regenerating liver. Am J Med Sci. 1994;307(3):167-172.

6. Tagashira M, Kitagawa T, Isonishi S, Okamoto A, Ochiai K, Ohtake Y. Mithramycin represses MDR1 gene expression in vitro, modulating multidrug resistance. Biol Pharm Bull. 2000;23(8):926-929.

7. Bianchi N, Osti F, Rutigliano C, et al. The DNA-binding drugs mithramycin and chromomycin are powerful inducers of erythroid differentiation of human K562 cells. Br J Haematol. 1999;104(2): 258-265.

8. Fibach E, Bianchi N, Borgatti M, Prus E, Gambari R. Mithramycin induces fetal hemoglobin production in normal and thalassemic human erythroid precursor cells. Blood. 2003;102(4):1276-1281.

9. Gambari R, Fibach E. Medicinal chemistry of fetal hemoglobin inducers for treatment of beta-thalassemia. Curr Med Chem. 2007;14: 199-212.

10. King PD, Perry MC. Hepatotoxicity of chemotherapy. Oncologist. 2001;6(12):162-176.

11. Fundueanu G, Nastruzzi C, Carpov A, Desbrieres J, Rinaudo M. Physico-chemical characterization of Ca-alginate microparticles produced with different methods. Biomaterials. 1999;20(15): 1427-1435.

12. Nastruzzi C, Cortesi R, Esposito E, et al. Liposomes as carriers for DNA-PNA hybrids. J Control Release. 2000;68(2):237-249.

13. Kedar U, Phutane P, Shidhaye S, Kadam V. Advances in polymeric micelles for drug delivery and tumor targeting. Nanomedicine. 2010; 6(6):714-729.

14. Nishiyama N, Kataoka K. Current state, achievements, and future prospects of polymeric micelles as nanocarriers for drug and gene delivery. Pharmacol Ther. 2006;112(3):630-648.

15. Bae Y, Nishiyama N, Fukushima S, Koyama H, Yasuhiro M, Kataoka K. Preparation and biological characterization of polymeric micelle drug carriers with intracellular $\mathrm{pH}$-triggered drug release property: tumor permeability, controlled subcellular drug distribution, and enhanced in vivo antitumor efficacy. Bioconjug Chem. 2005;16(1): $122-130$.

16. Vonarbourg A, Passirani C, Saulnier P, Benoit JP. Parameters influencing the stealthiness of colloidal drug delivery systems. Biomaterials. 2006; 27(24):4356-4373.

17. Letchford K, Burt H. A review of the formation and classification of amphiphilic block copolymer nanoparticulate structures: micelles, nanospheres, nanocapsules and polymersomes. Eur J Pharm Biopharm. 2007;65(3):259-269.

18. Park YJ, Lee JY, Chang YS, et al. Radioisotope carrying polyethylene oxide-polycaprolactone copolymer micelles for targetable bone imaging. Biomaterials. 2002;23(3):873-879.
19. Harada Y, Yamamoto T, Sakai M, et al. Effects of organic solvents on drug incorporation into polymeric carriers and morphological analyses of drug-incorporated polymeric micelles. Int J Pharm. 2011; 404(1-2):271-280.

20. Mikhail AS, Allen C. Block copolymer micelles for delivery of cancer therapy: transport at the whole body, tissue and cellular levels. J Control Release. 2009;138(3):214-223.

21. Gaucher G, Marchessault RH, Leroux JC. Polyester-based micelles and nanoparticles for the parenteral delivery of taxanes. J Control Release. 2010;143(1):2-12.

22. Barraud L, Merle P, Soma E, et al. Increase of doxorubicin sensitivity by doxorubicin-loading into nanoparticles for hepatocellular carcinoma cells in vitro and in vivo. J Hepatol. 2005;42(5):736-743.

23. Peer D, Karp J,Hong S, Farokhzad OC, Margalit R, Langer R. Nanocarriers as an emerging platform for cancer therapy. Nat Nanotechnol. 2007;2(12):751-760.

24. Danson S, Ferry D, Alakhov V, et al. Phase I dose escalation and pharmacokinetic study of pluronic polymer-bound doxorubicin (SP1049C) in patients with advanced cancer. Br J Cancer. 2004;90(11): 2085-2091.

25. Matsumura Y. Preclinical and clinical studies of anticancer drugincorporated polymeric micelles. J Drug Target. 2007;15(7-8): 507-517.

26. Cohen-Sela E, Teitlboim S, Chorny M, et al. Single and double emulsion manufacturing techniques of an amphiphilic drug in PLGA nanoparticles: formulations of mithramycin and bioactivity. $J$ Pharm Sci. 2009;98(4):1452-1462.

27. Bromberg L, Temchenko M. Solubilization of hydrophobic compounds by micellar solutions of hydrophobically modified polyelectrolytes. Langmuir. 1999; 15(25):8627-8632.

28. Gaucher G, Dufresne MH, Sant VP, Kang N, Maysinger D, Leroux JC. Block copolymer micelles: preparation, characterization and application in drug delivery. J Control Release. 2005;109(1-3):169-188.

29. Gaucher G, Satturwar P, Jones MC, Furtos A, Leroux JC. Polymeric micelles for oral drug delivery. Eur J Pharm Biopharm. 2010;762: $147-158$.

30. Karnik R, Gu F, Basto P, et al. Microfluidic platform for controlled synthesis of polymeric nanoparticles. Nano Lett. 2008;8(9): 2906-2912.

31. Alexis F, Pridgen E, Molnar LK, Farokhzad OC. Factors affecting the clearance and biodistribution of polymeric nanoparticles. Mol Pharm. 2008;5(4):505-515.

32. Jahn A, Stavis SM, Hong JS, Vreeland WN, DeVoe DL, Gaitan M. Microfluidic mixing and the formation of nanoscale lipid vesicles. ACS Nano. 2010;4(4):2077-2087.

33. Croy SR, Kwon GS. The effects of Pluronic block copolymers on the aggregation state of nystatin. J Control Release. 2004;95(2):161-171.

34. Mazzitelli S, Capretto L, Carugo D, Zhang X, Piva R, Nastruzzi C. Optimised production of multifunctional microfibres by microfluidic chip technology for tissue engineering applications. Lab Chip. 2011; 11(10):1776-1785.

35. Zhang X, Jones P, Haswell SJ. Attachment and detachment of living cells on modified microchannel surfaces in a microfluidic-based labon-a-chip system. Chem Eng J. 2008;135 Suppl 1:S82-S88.

36. Johnson BK. Flash nanoprecipitation of organic actives via confined micromixing and block copolymer stabilization [PhD thesis]. Princeton, $\mathrm{NJ}$ : Princeton University; 2003.

37. Tosi A, Mazzitelli S, Capretto L, Guerrieri R, Nastruzzi C. Optimization of lipospheres production by factorial design and their performances on a dielectrophoretic lab-on-a-chip platform. Colloids Surf A Physicochem Eng Asp. 2009;340(1):77-85.

38. Bohmer RM, Campbell TA, Bianchi DW. Selectively increased growth of fetal haemoglobin-expressing adult erythroid progenitors after brief treatment of early progenitors with transforming growth factor beta. Blood. 2000;95(9):2967-2974.

39. Rapoport N. Physical stimuli-responsive polymeric micelles for anticancer drug delivery. Prog Polym Sci. 2007;32(8-9):962-990. 
40. Johnson BK, Prud'homme RK. Mechanism for rapid self-assembly of block copolymer nanoparticles. Phys Rev Lett. 2003;91(11):118302.

41. Kabanov AV, Batrakova EV, Alakhov VY. Pluronic block copolymers as novel polymer therapeutics for drug and gene delivery. J Control Release. 2002;82(2-3):189-212.

42. Frey DD, Engelhardt F, Greitzer EM. A role for "one-factor-at-a-time" experimentation in parameter design. Res Eng Des. 2003;14(2): 65-74.

43. Frey DD, Wang H. Adaptive one-factor-at-a-time experimentation and expected value of improvement. Technometrics. 2006;48(3):418-431.

44. Capretto L, Carugo D, Cheng W, Hill M, Zhang X. Continuous-flow production of polymeric micelles in microreactors: experimental and computational analysis. J Colloid Interface Sci. 2011;357(1): 243-251.

45. Sezgin Z, Yüksel N, Baykara T. Preparation and characterization of polymeric micelles for solubilization of poorly soluble anticancer drugs. Eur J Pharm Biopharm. 2006;64(3):261-268.

46. Choucair A, Eisenberg A. Interfacial solubilization of model amphiphilic molecules in block copolymer micelles. J Am Chem Soc. 2003;125(39):11993-12000.

47. Elhasi S, Astaneh R, Lavasanifar A. Solubilization of an amphiphilic drug by poly(ethylene oxide)-block-poly(ester) micelles. Eur J Pharm Biopharm. 2007;65(3):406-413.

48. Allen C, Maysinger D, Eisenberg A. Nano-engineering block copolymer aggregates for drug delivery. Colloids Surf B Biointerfaces. 1999; 16(1-4):3-27.

49. Li SD, Huang L. Pharmacokinetics and biodistribution of nanoparticles. Mol Pharm. 2008;5(4):496-504.

50. Bromberg L. Polymeric micelles in oral chemotherapy. J Control Release. 2008;128(2):99-112.

51. Moghimi SM, Hunter AC, Murray JC. Long-circulating and targetspecific nanoparticles: theory to practice. Pharmacol Rev. 2001; 53(2):283-318.
52. Kwon GS. Polymeric micelles for delivery of poorly water-soluble compounds. Crit Rev Drug Ther Drug Carrier Syst. 2003;20(5): 357-403.

53. Bae Y, Diezi TA, Zhao A, Kwon GS. Mixed polymeric micelles for combination cancer chemotherapy through the concurrent delivery of multiple chemotherapeutic agents. $J$ Control Release. 2007;122(3):324-330.

54. Fordis CM, Anagnou NP, Dean A, Nienhuis AW, Schechter AN. A beta-globin gene, inactive in the K562 leukemic cell, functions normally in a heterologous expression system. Proc Natl Acad Sci US A. 1984;81(14):4485-4489.

55. Remsing LL, González AM, Nur-e-Alam M, et al. Mithramycin SK, a novel antitumor drug with improved therapeutic index, mithramycin SA, and demycarosyl-mithramycin SK: three new products generated in the mithramycin producer Streptomyces argillaceus through combinatorial biosynthesis. J Am Chem Soc. 2003;125(19):5745-5753.

56. Baig I, Perez M, Braña AF, et al. Mithramycin analogues generated by combinatorial biosynthesis show improved bioactivity. J Nat Prod. 2008;71(2):199-207.

57. McConnell SC, Huo Y, Liu S, Ryan TM. Human globin knock-in mice complete fetal-to-adult hemoglobin switching in postnatal development. Mol Cell Biol. 2011;31(4):876-883.

58. Jamsai D, Zaibak F, Vadolas J, et al. A humanized BAC transgenic/ knockout mouse model for HbE/beta-thalassemia. Genomics. 2006; 88(3):309-315.

59. Chakraborty H, Devi PG, Sarkar M, Dasgupta D. Multiple functions of generic drugs: future perspectives of aureolic acid group of anti-cancer antibiotics and non-steroidal anti-inflammatory drugs. Mini Rev Med Chem. 2008;8(4):331-349.

60. Bianchi N, Zuccato C, Lampronti I, Borgatti M, Gambari R. Expression of miR-210 during erythroid differentiation and induction of gammaglobin gene expression. BMB Rep. 2009;42(8):493-499.
International Journal of Nanomedicine

\section{Publish your work in this journal}

The International Journal of Nanomedicine is an international, peerreviewed journal focusing on the application of nanotechnology in diagnostics, therapeutics, and drug delivery systems throughout the biomedical field. This journal is indexed on PubMed Central, MedLine, CAS, SciSearch ${ }^{\circledR}$, Current Contents ${ }^{\circledR} /$ Clinical Medicine,

\section{Dovepress}

Journal Citation Reports/Science Edition, EMBase, Scopus and the Elsevier Bibliographic databases. The manuscript management system is completely online and includes a very quick and fair peer-review system, which is all easy to use. Visit http://www.dovepress.com/ testimonials.php to read real quotes from published authors. 